\title{
Regularity of Volume-Minimizing Graphs
}

\section{David L. Johnson 63 Penelope Smith}

Abstract. Let $M$ be a compact, oriented Riemannian $n$ manifold, and let $B \rightarrow M$ be a fiber bundle over $M$, with compact fiber $F$. Given a section of $B$, its volume is defined as the $n$-dimensional Hausdorff measure (or mass) of the graph of $\varphi$. We show that a volume-minimizing graph in a given homology class, which is in general a rectifiable section, is a continuous graph over all but a set of Hausdorff codimension 3 in the base $M$, unless the fiber $F$ admits stable 2-dimensional minimal currents (without boundary), in which case there could be a codimension 2 set of singular points with poles (support of the current over a singular point) consisting of such 2-dimensional currents. Locally, we also show that a volume-minimizing graph of a map $\varphi: \Omega \rightarrow F$ for $\Omega \subset \mathbb{R}^{n}$ with prescribed boundary $\varphi_{\mid \partial \Omega}=\psi: \partial \Omega \rightarrow F$ has codimension-3 poles in $\Omega$, under the assumption that $\Omega$ is convex and that $F$ is a compact, convex subset of Euclidean space.

This work grew out of a question posed by Herman Gluck and Wolfgang Ziller regarding the "volume of flows", volumes of sections of the unit tangent bundle of a compact manifold, viewed as one-dimensional foliations on $M$. They established that the standard Hopf fibration, as a foliation on $S^{3}$, minimizes volume among all such sections. However, in higher dimensions the Hopf fibrations are not volume-minimizing, and it is likely that volume-minimizing flows on these manifolds are singular, due to examples of Sharon Pedersen.

In this setting our results show that, except in dimension 3, outside of a codimension-3 set of possible "poles", a minimizing foliation will be $C^{1}$. In dimension 3 there can be a codimension- 2 set of poles, almost all of which involve the whole fiber in that for each unit tangent vector at almost-all poles (in the Hausdorff measure on the set of singular points), there is a limit of tangents of a leaf converging to that vector. 
Introduction. In [5], Herman Gluck and Wolfgang Ziller asked which one-dimensional, transversely oriented foliation $\mathcal{F}$ (called a flow) on an odddimensional round sphere is best-organized, in the sense that the image of the natural section $\xi: M \rightarrow T_{1}(M)$ of the unit tangent bundle, whose value at $x$ is the unit tangent vector of the leaf of $\mathcal{F}$ through $x$ consistent with the orientation of $\mathcal{F}$, has smallest $n$-dimensional Hausdorff measure. They were able to show that there is a three-form on $T_{1}\left(S^{3}\right)$ which calibrates the fibers of the Hopf fibrations on $S^{3}$, thus those foliations have the least volume of all such flows on the round three-sphere.

This question is a special case of a more general problem: Given a fiber bundle $\pi: B \rightarrow M$ with compact fiber $F$, which sections have the least volume? The volume is the $n$-dimensional Hausdorff measure of the image, or graph, of the section, where $n$ is the dimension of the base manifold. A natural metric on the bundle, compatible with the bundle structure and metrics on the base and the fiber, is assumed. In the special case of a trivial bundle, so that a section can be viewed as a map $\varphi: M \rightarrow F$, the volume of a section, which is the mass of the graph, is an energy functional with quite different properties from the energy of harmonic maps. R. Schoen, in [14], has shown that minimal graphs $\varphi: M \rightarrow N$ between surfaces $M$ and $N$, which he calls minimal maps, are graphs of conformal harmonic maps between the two surfaces, if the domain surface $M$ is complete and non-negatively curved. Example 3.5 below gives a sequence of examples of this type of minimal graph, leading to a singular minimal graph as a limiting case.

The three-sphere is, of course, a particularly nice manifold, and it would be too much to hope that such simple minima of this functional could always be obtained. Gluck and Ziller noted that their calibration did not extend to higherdimensional spheres, but they did not resolve the question of whether the higherdimensional Hopf fibrations were of minimal volume. Such would naively appear to be the case, since the Hopf fibrations appear to be extremely well-organized, and they clearly are critical points of the volume functional $\mathcal{V}(F):=\mathcal{H}^{n}\left(\xi_{F}(M)\right)$, where $\xi_{F}: M \rightarrow T_{1}(M)$ is the tangent section of $\mathcal{F}$ and $\mathcal{H}^{n}$ denotes Hausdorff $n$-dimensional measure. However, the first-named author showed in [7] that, in dimensions larger than 3, the Hopf fibrations do not define even local minima of the volume functional, and there are large families of deformations of the Hopf flows which have smaller volume.

In her thesis [12], Sharon Pedersen constructed a likely candidate for the minimum-volume flows on odd-dimensional round spheres of dimension at least 5. Her examples had lesser volume than the Hopf flows, and asymptotically (as the dimension grows) stay within a factor of 2 of a theoretical minimum determined by Gluck and Ziller. Their lower bound is determined by calibrating the cohomology class of twice that of graphs of flows in $T_{1}(M)$, for $M$ a round sphere. The difficulty, from a differential-geometric viewpoint, of Pedersen's examples is that they are singular. There is a single point on the base sphere over 
which her example is not a graph of a smooth section. Her minimizing "cycle" (or current) $P$ contains the entire fiber over this point, and the minimizing current itself has a single singular point. At regular points, $P$ is a minimal submanifold of the unit tangent bundle, and for dimensions at least 5 the singular point of the current $P$ has a tangent cone satisfying Gary Lawlor's angle criterion for minimality of a cone [9]. It is still an open question, however, whether the currents $P$ represent absolute minima for flows on round spheres.

The present work generalizes this situation to the question of minimizing the volume of sections of arbitrary fiber bundles with Riemannian metrics defined by a given connection, which of course includes the volume of flows as an important special case.

Let $M$ be an $n$-dimensional, compact, oriented Riemannian manifold, and let $B \rightarrow M$ be a fiber bundle over $M$, with fiber $F$ a compact, $j$-dimensional manifold which admits a smooth action of $G, G \times F \rightarrow F$, through isometries, where $G$ is the structure group of a principal bundle $P \rightarrow M$ to which $B$ is associated. Without loss of generality it may be assumed that $G$ is compact. According to [10], there is a Euclidean space $\mathbb{R}^{m}$ into which $F$ isometrically embeds, in such a fashion that the group $G$ acts by isometries, and the group action extends to a representation of $G$ on $\mathbb{R}^{m}$.

Remark. Earlier versions of this result included as an assumption that such an equivariant isometric embedding existed. The authors wish to thank Roger Schlafly for pointing out that such an embedding always exists.

Given a connection $\mathcal{H}$ on $P$, there is a unique Riemannian metric on $B$ so that the group acts by isometries, the fiber inclusions are isometric, and the horizontal distributions of the associated connection on $B$ are orthogonal to the fibers [13]. There is then a natural energy functional on the space of all sections of $F$, called the volume (or mass), defined to be the Hausdorff $n$-dimensional measure of the image or graph of the section.

The volume of a section $\sigma, \mathcal{V}(\sigma)$, is defined as the Hausdorff $n$-dimensional measure of the graph, $\mathcal{H}^{n}(\sigma(M))$. Replacing the set of graphs of sections by the larger class of $n$-dimensional integral currents homologous to some graph, geometric measure theory results [2] guarantee the existence of a minimizing current in $T_{1}(M)$ within each homology class. Moreover, such a minimizing current is regular outside of a singular set of codimension at least 2 on the current [1]. However, such a mass-minimizing current need not be a graph, nor in the closure of the space of graphs. A weak volume-minimizing (singular) section in a homology class is an integral current minimizing the volume functional in the closure of the set of graphs within that homology class, using the Cartesian norm, which will be defined below. We make no claim that the volume-minimizing section is the same as the mass-minimizing current in the homology class. In general, a volume-minimizing singular section is $M$-almost-everywhere a smooth 
map. Singularities of the section on $M$ result both from singularities of the current and points where the current fails to be transverse to the fibers of the bundle projection. Thus, regularity properties for minimizing sections are not necessarily those for volume-minimizing integral currents.

A result of Giaquinta, et al [3], slightly generalized to the case of nontrivial bundles, guarantees that a minimizing singular section is $M$-almost-everywhere a section. Furthermore, since the situation involves, locally, graphs of maps from $\mathbb{R}^{n}$ to $F$, the vertical direction is compact. These features are key to the proof of the following result, which is the main result of this paper:

Theorem 0.1. Let $M$ be a compact, oriented, Riemannian n-manifold, and let $B \rightarrow M$ be a fiber bundle with compact fiber $F$, with the Riemannian metric determined by a connection on an associated principal bundle.

(1) If $F$ does not contain a closed, stable, minimal two-dimensional rectifiable current, then, for any homology class of sections of $B$, there is a volumeminimizing representative with singular locus $P \subset M$ of codimension at least 3. Except for this singular locus, the graph defines a continuous section of $B_{\mid M-P}$.

(2) If $F$ does contain a closed, stable, minimal two-dimensional rectifiable current, then, for any homology class of sections of $B$, there is a volumeminimizing representative with singular locus $P$ of codimension at least 2 in $M$. If there is a codimension 2 set of critical points, the support of the graph meets $P$-almost-all fibers over singular points in a closed, stable, minimal, rectifiable 2-dimensional current, where the measure on $P$ is Hausdorff $(n-2)$-dimensional measure.

Remark. We say that a point $x \in M$ is a pole point of a section $S$, where $B$ is an $F$-bundle, if it is a singular point of the section $S$, that is, a place where the current meets the fiber in more than one point. Here the set $P$ is the set of pole points. The graph will be continuous outside of $P$. We specifically exclude from the definition of a pole point a place where a minimizing current meets a fiber at only one point, but whose tangent cone is not transverse to that of the fiber. Thus the graph $y=x^{1 / 3}$ will not be considered to have a pole over 0 .

The main result is only a slight generalization to bundles of the following local statement, which is most-easily stated when the fiber $F$ is a compact, convex subset of Euclidean space $\mathbb{R}^{j}$.

Theorem 0.2. Let $\Omega$ be a bounded, convex domain in $\mathbb{R}^{n}$, and let $\psi$ : $\partial \Omega \rightarrow F$ be a continuous map almost-everywhere with respect to Hausdorff ( $n-$ $1)$-dimensional measure on $\partial \Omega$, such that the support of the graph $\operatorname{graph}(\psi) \subset$ $\partial \Omega \times F$ is rectifiable. Then, there is a volume-minimizing graph $\varphi: \Omega \rightarrow F$ continuous except for a singular locus $P \subset \Omega$ of codimension at least 3 in $\Omega$, with $\varphi_{\mid \partial \Omega}=\psi$. 
The following is clearly a simple corollary of Theorem 0.1 , but is stated separately since the question it answers was the original motivation for this work.

Corollary 0.3. Let $M$ be a compact, oriented, Riemannian manifold. Then, for any homology class of one-dimensional foliations on $M$, there is a volume-minimizing representative with singular locus $P \subset M$ of codimension at least 3, except perhaps if $\operatorname{dim}(M)=3$. If $\operatorname{dim}(M)=3$, there may be a codimension 2 set of singular points $P \subset M$. The minimizing singular foliation will be $C^{1}$ outside of the singular set. In dimension 3, P-almost-all singular points are poles with the entire fiber as the support of the pole, where the measure on $P$ is the Hausdorff $(n-2)$-dimensional measure.

Given a section $\xi$ of $B \rightarrow M$, the volume $\mathcal{V}(\xi)$ can be evaluated by:

$$
\mathcal{V}(\xi)=\int_{M} \sqrt{1+(\nabla \xi)^{2}+\cdots+\underbrace{(\nabla \xi \wedge \cdots \wedge \nabla \xi)^{2}}_{j}} d V_{M}
$$

where the vector wedge is interpreted by $\nabla \alpha \wedge \nabla \beta(X, Y):=\frac{1}{2}\left(\nabla_{X} \alpha \wedge \nabla_{Y} \beta-\right.$ $\left.\nabla_{Y} \alpha \wedge \nabla_{X} \beta\right)$, etc., so that $(\nabla \xi)^{\wedge k}\left(X_{1}, \ldots, X_{k}\right)=\nabla_{X_{1}} \xi \wedge \cdots \wedge \nabla_{X_{j}} \xi$. The sum is taken over wedges of order up to $j$, rather than $n$, since the tangent space to the fiber $F_{x}$ at $\xi$ is $j$-dimensional.

As indicated above, however, there is still some work left to show, even in low dimensions, that the set of points on $M$ at which the section is not smooth, the singular locus $P$ of the minimizer, is as small as claimed in Theorem 0.1. In order to examine the character of a generic point $x \in P$, we first restrict to a small disk in $M$. The support of the volume-minimizing section $S$ will be shown to be, following Theorem 1.2, a smooth graph $u=\xi(x)$ for $M$-almost-all $x \in M$. Let $B_{r}$ be the metric ball in $M$ centered at $x_{0}$ with radius $r$, where a priori we presume that $x_{0} \in P$ is in the singular locus. Denote by $S_{r}$ the restriction $S_{r}:=S\left\llcorner\pi^{-1}\left(B_{r}\right)\right.$ to the cylinder of radius $r, \pi^{-1}\left(B_{r}\right):=C_{r}$, and set $T_{r}:=\partial S_{r}$. For almost-all $r$ (in the Lebesgue measure on $\mathbb{R}$ ), $T_{r}$ will also be rectifiable (by slicing theory [11], [2]), and will have finite mass, as can be seen by computing the measure of $S$ via integration over the base, using polar coordinates. Thus, for almost all $r, S_{r}$ will be an integral current. Set $Z:=\pi^{-1}\left(x_{0}\right) \cap \operatorname{Supp}(S)$.

We would like to thank the referee of the first version of this paper for many helpful suggestions. 
1. Rectifiable Sections. Giaquinta, Modica, and Souček consider in [3], [4], Cartesian currents, currents in a product $\Omega \times \mathbb{R}^{m}$ for $\Omega \subset \mathbb{R}^{n}$ a bounded domain which are the geometric-measure-theoretic analogues of graphs of maps $u: \Omega \rightarrow \mathbb{R}^{m}$. We recall the relevant portions of their work.

If $u: \Omega \rightarrow \mathbb{R}^{m}$ is a smooth map, the associated current is denoted by $T_{u}$. The components of a current $T$ are most easily defined for the smooth case, $T=T_{u}$, by

$$
\left[\left[T_{u}\right]_{\alpha \beta}(\varphi)=\sigma(\alpha, \bar{\alpha}) \int_{\Omega} \varphi(x, u(x)) M_{\beta \bar{\alpha}}(D u(x)) d x\right.
$$

for $\varphi \in C_{c}^{\infty}\left(\Omega \times \mathbb{R}^{m}\right)$. $\alpha$ and $\beta$ are multiindices with $\alpha=\left(\alpha_{1}, \ldots, \alpha_{p}\right)$, with $1 \leq \alpha_{1}<\cdots<\alpha_{p} \leq n,|\alpha|:=p, \bar{\alpha}$ the complement of $\alpha$, and, if $\alpha$ is null, that is, $|\alpha|=0$, then $\alpha:=0 . \sigma(\alpha, \bar{\alpha})$ is the sign of the shuffle permutation $(\alpha, \bar{\alpha})$. Then, in the preceding, $|\alpha|+|\beta|=n, D u(x)$ is the Jacobian matrix of $u$ at $x$, and $M_{\beta \bar{\alpha}}(D u(x))$ is the determinant of the Jacobian submatrix with rows $\beta$ and columns $\bar{\alpha}$. This definition can then be extended to the weak closure of the set of smooth graphs. In particular, if $\alpha=\beta=0,\left[\left[T_{u}\right]_{\overline{0} 0}(\varphi)=\int_{\Omega} \varphi(x, u(x)) d x\right.$. The volume functional can be re-cast into this language; for if $u: \Omega \rightarrow \mathbb{R}^{m}$ is smooth,

$$
\mathcal{V}(\operatorname{graph}(u))=M\left(T_{u}\right)=\int_{\Omega}\left\{\sum_{|\alpha|+|\beta|=n}\left(M_{\beta \bar{\alpha}}(D u)\right)^{2}\right\}^{1 / 2} d \operatorname{Vol}_{M}
$$

The Cartesian norm $\|T\|_{C}$ of $n$-dimensional currents $T$ in $\Omega \times \mathbb{R}^{N}$ is defined by $\|T\|_{C}=M(T)+\|T\|_{L^{1}(\Omega)}$, where $\|T\|_{L^{1}(\Omega)}:=\sup \left\{\left[[T]_{\overline{0} 0}(|y| \varphi(x, y))\right.\right.$ s.t. $\left.\|\varphi\|_{\infty} \leq 1\right\}$, and the supremum is taken over the set $\left\{\varphi(x, y), \varphi: \Omega \times \mathbb{R}^{m} \rightarrow \mathbb{R}\right.$ with $\left.\|\varphi\|_{\infty} \leq 1\right\}$.

The set $\operatorname{cart}\left(\Omega, \mathbb{R}^{m}\right)$ of Cartesian currents is defined by: $\operatorname{cart}\left(\Omega, \mathbb{R}^{m}\right):=$ $\left\{T \mid T\right.$ is a rectifiable current in $\Omega \times \mathbb{R}^{m}, \partial T=0$ in $\Omega \times \mathbb{R}^{m}, \pi_{*}(T)=\llbracket[\Omega] \in$ $\left.H^{n}(\Omega, \partial \Omega), T_{\overline{0} 0} \geq 0\right\}$, and $\operatorname{Cart}\left(\Omega, \mathbb{R}^{m}\right)$ is defined by $\operatorname{Cart}\left(\Omega, \mathbb{R}^{m}\right):=\{T \in$ $\operatorname{cart}\left(\Omega, \mathbb{R}^{m}\right): \exists u_{k} \in C^{1}\left(\Omega, \mathbb{R}^{m}\right)$, with $\sup _{k}\left\|T_{u_{k}}\right\|_{C}<\infty$ and $\left.T_{u_{k}} \rightarrow T\right\}$, which is closed with respect to weak convergence of currents with equibounded Cartesian norms.

Analogously, we define rectifiable sections $T$ in a rank- $k$ vector bundle $E \rightarrow M$, where $E$ has a smooth inner product $\langle$,$\rangle on the fibers. The inner$ product defines a collection of connections, called metric connections, which are compatible with the metric. Let a metric connection $\nabla$ be chosen. The connection $\nabla$ defines a Riemannian metric on the total space $E$ so that the projection $\pi: E \rightarrow M$ is a Riemannian submersion and so that the fibers are totally geodesic and isometric with the inner product space $E_{x} \equiv \mathbb{R}^{k}$, which has the 
original inner product [13], [8]. A rectifiable section $T \in \tilde{\Gamma}(E)$ is a rectifiable current in the weak closure (in the flat norm) of the set of $C^{1}$-sections of $E$. The components of $T \in \tilde{\Gamma}(E)$ are first defined for a smooth section $u \in \Gamma(E)$ by $\left[T_{u} \rrbracket_{\alpha \beta}(\varphi)=\sigma(\alpha, \bar{\alpha}) \int_{M} \varphi(x, u(x)) M_{\beta \bar{\alpha}}(\nabla u(x)) d x\right.$ for $\varphi \in C_{c}^{\infty}(E)$, where, as before, $M_{\beta \bar{\alpha}}(\nabla u(x))$ is the determinant of the $|\beta| \times|\beta|$ minor of $\nabla u$ with rows given by the multi-index $\beta$ and columns by $\bar{\alpha}$, with $|\beta|+|\alpha|=n$. An orthonormal basis $\left\{e_{i}\right\}$ of $T_{*}(M, x)$ and $\left\{v_{j}\right\}$ of $E_{x}$ is chosen locally, varying smoothly with $x$. The components of $T$ depend on this local choice of bases; if $\bar{\alpha}=\left(i_{1}, \ldots, i_{\ell}\right)$, $\beta=\left(j_{1}, \ldots, j_{\ell}\right)$, then $M_{\beta \bar{\alpha}}(\nabla u(x))=\operatorname{det}\left(\left\langle\nabla_{e_{i s}} u, v_{j_{t}}\right\rangle\right)$. This definition is then completed to all rectifiable sections. The Cartesian norm \|\|$_{C}$ can be defined for rectifiable sections as well, by $\|T\|_{C}=M(T)+\|T\|_{L^{1}(M)}$, where $M(T)$ is the mass (or volume) of $T$ and $\|T\|_{L^{1}(\Omega)}:=\sup \left\{[T]_{\overline{0} 0}\left(\|v\|_{E} \varphi(v)\right)\right.$ s.t. $\left.\|\varphi\|_{\infty} \leq 1\right\}$, with the supremum taken over all $\varphi(v), \varphi: E \rightarrow \mathbb{R}$ with $\|\varphi\|_{\infty} \leq 1$. Again following [3], define the space $\operatorname{cart}(E):=\{T \mid T$ is a rectifiable section in $E, \partial T=0$, $\left.\pi_{*}(T)=[[\Omega]] \in H^{n}(M), T_{\overline{0} 0} \geq 0\right\}$, and $\operatorname{Cart}(E):=\left\{T \in \operatorname{cart}(E): \exists u_{k} \in C^{1}(E)\right.$ with $\sup _{k}\left\|T_{u_{k}}\right\|_{C}<\infty$ and $\left.T_{u_{k}} \rightarrow T\right\}$, where $C^{1}(E)$ is the space of continuously differentiable sections of the vector bundle $E$. The same proof as in [3], using the Federer-Fleming closure theorem, shows the following compactness result.

Proposition $1.1([3])$. Let $\left\{T_{j}\right\} \subset \operatorname{cart}(E)$ (resp., $\left.\operatorname{Cart}(E)\right)$ be a sequence with equibounded Cartesian norm. Then, there is a subsequence which converges weakly to a current $T$ in $\operatorname{cart}(E)$ (resp., $\operatorname{Cart}(E))$.

Thus, given any weakly closed set of currents $A \subset \operatorname{Cart}(E)$, if $\|T\|_{L^{1}(M)}$ is bounded, a minimal mass element of $A$ will always exist in $\operatorname{Cart}(E)$.

Consider now a fiber bundle $B \rightarrow M$, with fiber $F$ a compact, $j$-dimensional manifold which admits a smooth action of $G, G \times F \rightarrow F$, through isometries, where $G$ is the structure group of a principal bundle $P \rightarrow M$ to which $B$ is associated. Without loss of generality it may be assumed that $G$ is compact. According to [10], there is a Euclidean space $\mathbb{R}^{m}$ into which $F$ isometrically embeds, in such a fashion that the group $G$ acts by isometries, and the group action extends to a representation of $G$ on $\mathbb{R}^{m}$. Given a connection $\mathcal{H}$ on $P$, there is a unique Riemannian metric on $B$ so that the group acts by isometries, the fiber inclusions are isometric, and the horizontal distributions of the associated connection on $B$ are orthogonal to the fibers [13].

Rectifiable sections $T$ of $B, T \in \tilde{\Gamma}(B)$, are rectifiable sections of $E$ whose support lies within $B$, which is a weakly closed condition, $\operatorname{cart}(B):=\operatorname{cart}(E) \cap$ $\tilde{\Gamma}(B)$, and $\operatorname{Cart}(B):=\operatorname{Cart}(E) \cap \tilde{\Gamma}(B)$. Since the fibers of $B$ are compact, as is the base manifold $M$, minimal-mass elements will exist in $\operatorname{cart}(B)$ or $\operatorname{Cart}(B)$, and mass-minimizing sequences within any path-component will have convergent subsequences in $\operatorname{cart}(B)$ or $\operatorname{Cart}(B)$. This follows from the definition of the 
Cartesian norm, which in this case is uniformly bounded by the mass of the current, since the fiber is compact. Since $\operatorname{Cart}(B)$ deals directly with weak limits of $C^{1}$ sections, we will consider that space in the remainder of this paper.

Theorem 1.2. Let $B$ be as above. A mass-minimizing element $S$ of $\operatorname{cart}(B)($ resp., $\operatorname{Cart}(B))$ has support which is a $C^{1, \alpha}$ graph over an open, dense subset of $M$, for all $0<\alpha<1$.

Proof. Since $S$ is rectifiable, it has density 1 over $M$-a.e. points in the base $M[3$, Lemma 1]. It also has a tangent plane at almost every point [11, 3.12]. However, except at those points where the tangent plane fails to be transverse to the fibers, which by [3, Theorem 5] projects to a set of measure 0 in $M$, the weak mean curvature [6] will necessarily be zero. The weak mean curvature $H$ at a point $p$ is defined in terms of the divergence with respect to a vector field $X$,

$$
\int_{S\llcorner U} \operatorname{div}_{S}(X) d \mathcal{H}^{n}=\int_{S\llcorner U} \nabla \cdot X d \mathcal{H}^{n}=-\int_{S\llcorner U} H \cdot X d \mathcal{H}^{n}
$$

for all neighborhoods $U$ of $p$ and for all vector fields $X$ so that $\operatorname{Supp}(X) \subset U$. The vector fields $X$, restricted to $S$, are tangents to variations of $S$. Minimality of $S$ among rectifiable sections will imply that $S$ is stationary with respect to variations which are variations among sections. However, in a small neighborhood of a point which is transverse to the fiber, any variation will infinitesimally be a variation through sections. Thus, at any such point, $H=0$.

Allard's theorem [6] now can be applied to show that there is a $\mathcal{L}^{n}$-measure 0 subset $Q \subset M$ over which $S$ is not transverse to the fibers, and that the regular points are the points of $S$ over $M \backslash Q$. That is, $S$ is the graph of some $C^{1, \alpha}$-section $u:(M \backslash Q) \rightarrow E_{\mid(M \backslash Q)}$. This follows since, away from the closed set $Q$, there will always be a neighborhood $U$ over which the mean curvature will vanish and the height (diameter of the image $\left(\Pi_{2}\right)_{\#}(S\llcorner U)$ on $F$ ) of $S\llcorner U$ is small enough, perhaps after a rotation of coordinates, to apply Allard's result [6]. Here $\Pi_{2}: \pi^{-1}(U) \rightarrow F$ is the projection onto the second factor.

Note. Theorem 1.2 does not imply that $S$ is a regular graph $S$-almost everywhere in the Hausdorff $n$-dimensional measure on $S$. The distinction is that there may be a positive-mass region of $S$ which projects to a set of Hausdorff $n$-dimensional measure 0 on the base, as illustrated in Figure 1.1.

For the next result, which will be a version of the maximum principle, $B$ will be assumed to be the trivial $F$-bundle over a ball of radius $r, F_{r}=$ $B_{r} \times F$. However, the results of this section can be extended locally to a general bundle over a compact Riemannian manifold $M$, with respect to a specific local trivialization, in the following way. Recall that the metric we use on the total space of such a bundle depends on a choice of connection on $B$. Locally, for some 
FiguRE 1.1: Graph with pole elements of positive measure

geodesic ball $B_{r} \subseteq M, B_{\mid B_{r}} \rightarrow B_{r}$ is trivial, $B_{\mid B_{r}} \simeq B_{r} \times F$. Let 0 denote the center of the geodesic ball $B_{r}$ in a geodesic normal coordinate system, and use the coordinate system along with the connection to define a specific trivialization of $B_{\mid B_{r}}$. Specifically, lift the geodesics emanating from 0 to horizontal curves in $B_{\mid B_{r}}$ for the associated connection (distribution) $H_{\nabla} \subset T_{*}(B)$. These horizontal lifts define a projection of $B_{\mid B_{r}}$ onto the fiber over 0 , by mapping $y \in B_{\mid B_{r}}$ to $\gamma(0)$, if $\gamma$ is the lift of the geodesic $\bar{\gamma}$ on $B_{r}$ with $\bar{\gamma}(0)=0$ and $\gamma(1)=y$. This projection then defines a specific trivialization of $B_{\mid B_{r}}$.

Let $S_{r}$ be a volume-minimizing rectifiable section in $\operatorname{Cart}\left(B_{r} \times F\right)$ with specified boundary values $S_{\mid \partial B_{r}}=T_{r}$. For an open dense subset $B_{r} \backslash\left(Q \cap B_{r}\right)$ of $B_{r}, S\left\llcorner\pi^{-1}\left(B_{r} \backslash\left(Q \cap B_{r}\right)\right)\right.$ can be viewed as the graph of a smooth map $u:\left(B \backslash\left(Q \cap B_{r}\right)\right) \rightarrow F$.

Proposition 1.3. $S_{r}$ satisfies the following maximum principle. Let $v \in$ $\Pi_{2}\left(\operatorname{Supp}\left(S_{r}\right)\right) \subset F$, where $\Pi_{2}: B_{r} \times F \rightarrow F$ is the projection onto the second factor. Assume that, for some $U \subset B_{r}, \Pi_{2}\left(\operatorname{Supp}\left(S_{r}\right) \cap \pi^{-1}(U)\right)$ is contained in a geodesic ball $W \subset F$ centered at $v$, which is geodesically convex. Then, any local maximum of the distance from $v$ must lie in $\Pi_{2}\left(\operatorname{Supp}\left(S_{r}\right) \cap \pi^{-1}(\partial U)\right)$.

Proof. Presume that $u_{0} \in \Pi_{2}\left(S_{r}\right)$ is an interior point (not on $\Pi_{2}\left(S_{r} \cap\right.$ $\left.\left.\pi^{-1}(\partial U)\right)\right)$ for which $\Pi_{2}\left(u_{0}\right)$ is farthest from $v$.

Presume for the moment that $S_{r}$ is the graph of some mapping $u: B_{r} \rightarrow F$. Write $u(x)$ in terms of $\delta$, that is: in terms of local Riemann polar coordinates on $F$, (the geodesic polar coordinate system derived from a Riemann normal coordinate system centered at $v): u(x)=\varphi^{-1}(\cos (\delta(x)) v+\sin (\delta(x)) a(x))$ for some map $a: B_{r} \rightarrow S^{j-1}$, where $\delta(x):=\operatorname{dist}\left(v, \Pi_{2}(x)\right)$ and $\varphi$ is a Riemann 
normal coordinate chart. This decomposes $u$ according to polar coordinates $y=(\delta, a)$ in the exponential image $u(x)=\operatorname{Exp}_{v}(y)=\varphi^{-1}(y)$, for $y \in T_{*}(F, v)$. This construction easily extends to the general case, where $S_{r}$ is an arbitrary Cartesian current, by considering the graphs of $\delta$ and $a$ as Cartesian currents as well.

Since the radial directions are perpendicular to the spherical images corresponding to constant values of $\delta$, we can write the functional for the volume of the graph, $\mathcal{V}(\operatorname{graph}(u))$, in terms of $\delta$ as well:

$$
\begin{aligned}
\mathcal{V}(\operatorname{graph}(u)) & =\int_{B_{r}} \sqrt{\sum_{\ell \geq 0}\left\|\nabla u^{\wedge \ell}\right\|^{2}} d V \\
& =\int_{B_{r}} \sqrt{\sum_{\ell \geq 0}\left\|(-\sin (\delta(x)) v+\cos (\delta(x)) a(x)) d \delta+\sin ((\delta) \nabla a)^{\wedge \ell}\right\|^{2}} d V \\
& =\int_{B_{r}} \sqrt{\sum_{\ell \geq 0}\left\|d \delta \wedge(\sin (\delta) \nabla a)^{\wedge \ell-1}+(\sin (\delta) \nabla a)^{\wedge \ell}\right\|^{2}} d V \\
& =\int_{B_{r}} \sqrt{\sum_{\ell \geq 0}^{j-1} \sin ^{2 \ell}(\delta)\left\|(1+d \delta) \wedge(\nabla a)^{\wedge \ell}\right\|^{2}} d V,
\end{aligned}
$$

where the vector wedge with $(-\sin (\delta(x)) v+\cos (\delta(x)) a(x)) d \delta$ can be replaced by the scalar wedge with $d \delta$ since $\sin (\delta(x)) v+\cos (\delta(x)) a(x)$ is orthogonal to $\nabla a$, since $v$ and $a(x)$ are orthogonal to $\nabla a$.

The hypotheses imply that there is a $\rho>0$ so that $\delta(x) \geq \rho$ only in the interior of $U$. Define $\hat{u}(x):=-\cos (\hat{\delta}(x)) v+\sin (\hat{\delta}(x)) a(x)$, where

$$
\hat{\delta}(x):= \begin{cases}\delta(x), & \text { if } \delta(x) \leq \rho, \\ \rho, & \text { if } \delta(x)>\rho .\end{cases}
$$

Since the geodesic ball $W_{\rho}$ of radius $\rho$ is convex, mass is decreased by projection onto $B_{R} \times \partial W_{\rho}$ of any graph with boundary contained in $B_{R} \times \partial W_{\rho}$, and with the rest of the graph outside of that ball. Thus,

$$
\begin{aligned}
\mathcal{V}(\operatorname{graph}(\hat{u})) & =\int_{B_{r}} \sqrt{\sum_{\ell=0}^{j-1} \sin ^{2 \ell}(\hat{\delta})\left\|(1+d \hat{\delta}) \wedge(\nabla a)^{\wedge \ell}\right\|^{2}} d V \\
& \leq \int_{B_{r}} \sqrt{\sum_{\ell=0}^{j-1} \sin ^{2 \ell}(\delta)\left\|(1+d \delta) \wedge(\nabla a)^{\wedge \ell}\right\|^{2}} d V=\mathcal{V}(\operatorname{graph}(u)) .
\end{aligned}
$$


However, $\delta(x)>\rho$ on a subset of $S_{r}$ with positive $S_{r}$-Hausdorff measure, since $S$ is an $n$-dimensional rectifiable current, and the set where $\delta(x)>0$ is open. Thus, the Lebesgue measure of the set on which the first integrand is strictly less than the second is positive. Hence the inequality above must be strict, contradicting the minimiality of $u$. This implies that the presumption of an interior maximum of $\delta$ must be false.

2. Basic Monotonicity Estimates. In this section, we consider a volumeminimizing rectifiable section in $\operatorname{Cart}\left(B_{r_{0}}^{n} \times F\right)$, where $j<n$, subject to the boundary conditions $\partial\left(S\left\llcorner\left(B_{r_{0}}^{n} \times F\right)\right)=T_{r_{0}}\right.$. Let $S_{r}:=S\left\llcorner\pi^{-1}\left(B_{r}\right)\right.$ for $r<r_{0}$. The results of this section will establish basic growth estimates on the volume of such a current in terms of the dimension of the intersection of the support of $S$ with the singular fiber over 0 . Let $Z:=\Pi_{2}\left(\operatorname{Supp}(S) \cap \pi^{-1}(0)\right)$ denote this singular fiber.

Proposition 2.1. There is a constant $A=A\left(r_{0}, T_{r_{0}}\right)$ so that $\mathcal{V}\left(S_{r}\right) \leq$ $A r^{n-j}$.

Proof. By the results of the previous section, there is a smooth mapping $u:\left(B_{r} \backslash Q\right) \rightarrow F$ whose graph is $S$ except over a set $Q \subseteq B_{r}$ of measure 0 . We will view $S$ as the graph of $u$, omitting reference to the singular set $Q$ except where it becomes relevant. At a fixed value $r$,

$$
\begin{aligned}
\mathcal{V}\left(S_{r}\right) & =\mathcal{V}\left(\operatorname{Graph}\left(u_{\mid B_{r}}\right)\right)=\mathcal{V}(u, r) \\
& =\int_{B_{r}} \sqrt{1+\|\nabla u\|^{2}+\cdots+\left\|\nabla u^{\wedge j}\right\|^{2}} d V \\
& =\int_{0}^{r} \int_{S^{n-1}} \sqrt{1+\|\nabla u\|^{2}+\cdots+\left\|\nabla u^{\wedge j}\right\|^{2}} \rho^{n-1} d V_{S^{n-1}} d \rho,
\end{aligned}
$$

in polar coordinates.

Replace $u$ in $B_{r}$ by the radially-invariant $v$ defined by $v(x):=u(r x /\|x\|)$, which in polar coordinates becomes $v(\rho, \theta)=u(r, \theta)$. Note that, for a Cartesian coordinate $x^{i}$ orthogonal to the radial direction, $\partial u / \partial x^{i}=(1 / r) \partial u / \partial \theta^{i}$, where $\theta^{i}$ is a corresponding (unit) coordinate on the unit $(n-1)$-sphere and $\nabla_{\theta}$ is the total covariant differential on that sphere. In particular, $\nabla u=\partial u / \partial \rho d \rho+(1 / \rho) \nabla_{\theta} u$ and $\nabla v=(1 / \rho) \nabla_{\theta} v$. This implies, for $\rho \leq r$, 


$$
\begin{aligned}
& \mathcal{V}(u, r) \leq \mathcal{V}(v, r) \\
& =\int_{0}^{r} \int_{S^{n-1}} \sqrt{1+\frac{1}{\rho^{2}}\left\|\nabla_{\theta} v\right\|^{2}+\cdots+\frac{1}{\rho^{2 j}}\left\|\nabla_{\theta} v^{\wedge j}\right\|^{2} \rho^{n-1}} d V_{S^{n-1}} d \rho \\
& =\int_{0}^{r} \int_{S^{n-1}} \sqrt{\rho^{2 j}+\rho^{2(j-1)}\left\|\nabla_{\theta} v\right\|^{2}+\cdots+\left\|\nabla_{\theta} v^{\wedge j}\right\|^{2}} \rho^{n-1-j} d V_{S^{n-1}} d \rho \\
& \leq \int_{0}^{r} \int_{S^{n-1}} \sqrt{r^{2 j}+r^{2(j-1)}\left\|\nabla_{\theta} v\right\|^{2}+\cdots+\left\|d_{\theta} v^{\wedge j}\right\|^{2}} r^{n-1-j} d V_{S^{n-1}} \frac{\rho^{n-1-j}}{r^{n-1-j}} d \rho \\
& =\int_{S^{n-1}} \sqrt{r^{2 j}+r^{2(j-1)}\left\|\nabla_{\theta} v\right\|^{2}+\cdots+\left\|d_{\theta} v^{\wedge j}\right\|^{2}} r^{n-1-j} d V_{S^{n-1}} \int_{0}^{r} \frac{\rho^{n-1-j}}{r^{n-1-j}} d \rho \\
& =\frac{\partial}{\partial r}(\mathcal{V}(v, r)) \int_{0}^{r} \frac{\rho^{n-1-j}}{r^{n-1-j}} d \rho \\
& \leq \frac{\partial}{\partial r}(\mathcal{V}(u, r)) \int_{0}^{r} \frac{\rho^{n-1-j}}{r^{n-1-j}} d \rho \frac{\partial}{\partial r}(\mathcal{V}(u, r)) \cdot \leq \frac{r}{n-j}
\end{aligned}
$$

Figure 2.1: Graph OF $u$

The inequality $\partial / \partial r(\mathcal{V}(v, r)) \leq \partial / \partial r(\mathcal{V}(u, r))$ follows from:

$$
\begin{aligned}
\frac{\partial}{\partial r}(\mathcal{V}(v, r)) & =\int_{S^{n-1}} \sqrt{r^{2 j}+r^{2(j-1)}\left\|\nabla_{\theta} v\right\|^{2}+\cdots+\left\|d_{\theta} v^{\wedge j}\right\|^{2}} r^{n-1-j} d V_{S^{n-1}} \\
& =\int_{S^{n-1}} \sqrt{r^{2 j}+r^{2(j-1)}\left\|\nabla_{\theta} u\right\|^{2}+\cdots+\left\|d_{\theta} u^{\wedge j}\right\|^{2}} r^{n-1-j} d V_{S^{n-1}}
\end{aligned}
$$


Since $\rho=r$, we have:

$$
\begin{aligned}
\frac{\partial}{\partial r}(\mathcal{V}(v, r)) & \leq \int_{S^{n-1}} \sqrt{1+\|d u\|^{2}+\cdots+\left\|d u^{\wedge j}\right\|^{2}} r^{n-1} d V_{S^{n-1}} \\
& =\frac{\partial}{\partial r}(\mathcal{V}(u, r)) .
\end{aligned}
$$

The derivative $\partial / \partial r(\mathcal{V}(u, r))$ exists for almost every $r$ since the function is increasing as a function of $r$.

Figure 2.2: GRAPH OF $v$

The argument then proceeds in a standard fashion [11]. Since, for $\mathcal{L}^{1}$ a.e. $r$,

$$
\mathcal{V}(u, r) \leq\left(\frac{r}{n-j}\right) \frac{\partial}{\partial r}(\mathcal{V}(u, r))
$$

and $\mathcal{V}(u, r)$ is increasing, $\mathcal{V}(u, r) / r^{n-j}$ is also increasing. Thus, if $A:=$ $\mathcal{V}\left(u, r_{0}\right) / r_{0}^{n-j}, \mathcal{V}(u, r) \leq A r^{n-j}$, for all $r \leq r_{0}$.

Remark. The local version of Pedersen's example, where $j=n-1$, exhibits precisely this growth rate, at the point over which the singularity occurs.

Proposition 2.2. Let $S$ be a volume-minimizing rectifiable section in $\operatorname{Cart}\left(B_{r_{0}}^{n} \times F\right)$, and presume that, for some $r>0$ the boundary currents $\partial\left(\left(\Pi_{2}\right)_{\#}\left(S\left\llcorner B_{r}^{n} \times F\right)\right)=\left(\Pi_{2}\right)_{\#} T_{r}\right.$ have support of dimension at most $k$ where $k$ is a nonnegative integer. Then, there is a number $A$ so that $\mathcal{V}\left(S_{r}\right) \leq A r^{n-k}$. 
Proof. As in Proposition 2.1, replace $u$ in $B_{r}$ by the radially-invariant map $v$ defined by $v(x):=u(r x /\|x\|)$, which in geodesic polar coordinates becomes $v(\rho, \theta)=u(r, \theta)$.

Let $x^{i}$ be a Cartesian coordinate orthogonal to the radial direction defined by $x^{i}=r \theta^{i}$, where $\theta^{i}$ is a corresponding (unit-speed) coordinate on the unit $(n-1)$-sphere and $\nabla_{\theta}$ is the total covariant differential on that sphere. Then, $\partial u / \partial x^{i}=(1 / r) \partial u / \partial \theta^{i}$. This implies, as before, that:

$$
\begin{aligned}
\mathcal{V}(u, r) & \leq \mathcal{V}(v, r) \\
& =\int_{0}^{r} \int_{S^{n-1}} \sqrt{1+\frac{1}{\rho^{2}}\left(\nabla_{\theta} v\right)^{2}+\cdots+\frac{1}{\rho^{2 j}}\left(\nabla_{\theta} v^{\wedge j}\right)^{2}} \rho^{n-1} d V_{S^{n-1}} d \rho \\
& =\int_{0}^{r} \int_{S^{n-1}} \sqrt{\rho^{2 j}+\cdots+\rho^{2(j-k)}\left(d_{\theta} v^{\wedge k}\right)^{2}+\cdots+\left(\nabla_{\theta} v^{\wedge j}\right)^{2}} \rho^{n-1-j} d V_{S^{n-1}} d \rho .
\end{aligned}
$$

At this point, note that the terms past the $k^{\text {th }}$ inside the radical are $\mathcal{L}^{n}$-almost everywhere 0 , by the hypotheses that the boundary has support of dimension at most $k$ in the fiber, since such terms would involve more than $k$ vertical dimensions. Thus,

$$
\begin{aligned}
& \mathcal{V}(u, r) \\
& \leq \int_{0}^{r} \int_{S^{n-1}} \sqrt{\rho^{2 k}+\rho^{2(k-1)}\left(d_{\theta} v\right)^{2}+\cdots+\left(\nabla_{\theta} v^{\wedge k}\right)^{2}} \rho^{n-k-1} d V_{S^{n-1}} d \rho \\
& \leq \int_{0}^{r} \int_{S^{n-1}} \sqrt{r^{2 k}+r^{2(k-1)}\left(\nabla_{\theta} v\right)^{2}+\cdots+\left(d_{\theta} v^{\wedge k}\right)^{2}} r^{n-k-1} d V_{S^{n-1}} \frac{\rho^{n-k-1}}{r^{n-k-1}} d \rho \\
& \leq \int_{0}^{r} \frac{d}{d r}(\mathcal{V}(v, r)) \frac{\rho^{n-k-1}}{r^{n-k-1}} d \rho \\
& \leq \int_{0}^{r} \frac{d}{d r}(\mathcal{V}(u, r)) \frac{\rho^{n-k-1}}{r^{n-k-1}} d \rho \\
& \leq \frac{r}{n-k} \cdot \frac{d}{d r}(\mathcal{V}(u, r)) .
\end{aligned}
$$

As before, this implies that there is an $A$ so that $\mathcal{V}(u, r) \leq A r^{n-k}$.

3. Horizontal tangent cones. Even locally the concept of tangent cones, being limits of (uniformly) stretched currents, loses the special nature of Cartesian sections of a fiber bundle. Such stretched currents are no longer in the class of sections of the bundle, and the information they contain can be no more than that given in general geometric measure theory. Our modification of that notion, called horizontal tangent cones, or $h$-cones, provides information specifically tailored to sections of bundles, and still provides most of the local geometric information of a full tangent cone. 
Definition. For $\lambda>0$, and $x_{0} \in B_{r}$, define $\nu_{x_{0}, \lambda}: B_{r} \times F \rightarrow B_{\lambda r} \times F$ by $\nu_{x_{0}, \lambda}(x, y)=\left(x_{0}+\lambda\left(x-x_{0}\right), y\right)$. Given a section $\varphi \in \Gamma\left(B_{r} \times F\right)$, (i.e., $\varphi$ : $B_{r} \rightarrow F$ ) where $B_{r} \subseteq \mathbb{R}^{n}$, and given $x_{0} \in B_{r}, \lambda>0$, define $\varphi_{x_{0}, \lambda}: B_{r} \rightarrow F$ by $\varphi_{x, \lambda}(x):=\varphi\left(x_{0}+\lambda\left(x-x_{0}\right)\right)=\varphi\left(\nu_{x_{0}, \lambda}(x)\right)$, for $x \in\left\{x \mid x_{0}+\lambda\left(x-x_{0}\right) \in B_{r}\right\}$. For $\lambda$ small enough and $x$ in the interior of $B_{r}, \varphi_{x_{0}, \lambda}$ is defined on all of $B_{r}$. Viewing these graphs as currents defines $\varphi_{x, \lambda}$ for all $\varphi \in \operatorname{Cart}\left(B_{r} \times F\right) . \varphi_{x, \lambda}$ will be called the $\lambda$-stretch of $\varphi$ at $x$, and will be denoted, following [2], by $\left(\nu_{x_{0}, 1 / \lambda}\right)_{\#}(S)$ or by $S_{x_{0}, \lambda}$, if the current is given as $S$. This is a slight abuse of notation, since $S_{x_{0}, \lambda}=\left(\nu_{x_{0}, 1 / \lambda}\right)_{\#}(S)\left\llcorner B_{r} \times F\right.$. To see that the $1 / \lambda$ is correct, rather than $\lambda$, note that the supports agree.

A horizontal cone, or $h$-cone, at $x_{0}$ is a $\psi \in \operatorname{Cart}(B \times F)$ so that $\psi_{x_{0}, \lambda}=\psi$.

Figure 3.1: A $h$-cone with an isolated pole point

The support of $\varphi_{x_{0}, \lambda}$ in $B_{r} \times F$ will clearly be $\nu_{x_{0}, 1 / \lambda}(\operatorname{Supp}(\varphi)) \cap B_{r} \times F$. Given a sequence $\lambda_{k} \rightarrow 0$, the horizontal tangent cone of $\varphi$ (or $h$-cone of $\varphi$ ) at $x_{0}$ associated to this sequence is an $h$-cone $\psi_{x_{0}} \in \operatorname{Cart}(B \times F)$ which is the limit in $\operatorname{Cart}\left(B_{r} \times F\right)$ of the sequence $\varphi_{k}:=\varphi_{x_{0}, \lambda_{k}}$. Just as for general tangent cones, we do not state that $h$-cones are uniquely determined by $\varphi$, but may depend upon the sequence of stretches.

According to the Compactness Theorem 1.1 (cf. [3]), if the Cartesian norms of $\varphi_{k}$ are uniformly bounded, for some subsequence of a given sequence $\lambda_{k} \rightarrow 0$, the associated limit will exist. Proving it to be an $h$-cone follows similarly to standard geometric measure theory.

In one sense, the information provided by $h$-cones is rather limited. If $x_{0}$ is a regular point of $\varphi$, then $\psi_{x_{0}}$ will be the graph of a constant. However, if 
$x_{0}$ is a singular point, then the $h$-cone associated to a sequence of stretches will have essentially the same singular behavior as $\varphi$, but will otherwise have a much simpler structure.

Definition. Let $P \subset B_{r}$ be the set of pole points, points in $B_{r}$ over which the fiber of the bundle meets $\operatorname{Supp}(S)$ more than once. The points $y \in \operatorname{Supp}(S) \cap$ $\pi^{-1}(x)$, where $x \in P$, will be called pole elements.

Theorem 3.1. Let $S \in \operatorname{Cart}\left(B_{r} \times F\right)$ minimize volume over all rectifiable Cartesian currents with the same boundary $T=S_{\mid \partial B_{r} \times F}$. Over each $x \in B_{r}$ there exists an h-cone.

Remark. By the compactness theorem, what needs to be shown is that the Cartesian norm of the stretched current $S_{x, \lambda}$ is bounded, independent of $\lambda$. This condition is trivially satisfied for points which are not pole points, since such points will have the horizontal plane through the current over that point as an $h$-cone.

Proof. Given an embedding of $F$ in some $\mathbb{R}^{m}$, an orthonormal coordinate system $\left\{y_{1}, \ldots, y_{m}\right\}$ on $\mathbb{R}^{m}$, and an orthonormal coordinate system $\left\{x_{1}, \ldots, x_{n}\right\}$ on $B(x, \varepsilon) \subset \mathbb{R}^{n}$, for each multi-index $\alpha=\left(i_{1}, \ldots, i_{n-f}, j_{1}, \ldots, j_{f}\right)$ with $1 \leq i_{1}<$ $\cdots<i_{n-f} \leq n$ and $1 \leq j_{1}<\cdots<j_{f} \leq m$ for some $f \leq \min (m, n)$, the $n$-plane $W_{\alpha}$ is the coordinate $n$-plane in $B(x, \varepsilon) \times \mathbb{R}^{m} x_{i_{1}} \wedge \cdots \wedge x_{i_{n-f}} \wedge y_{j_{1}} \wedge \cdots \wedge y_{j_{f}}$, which has $f$ vertical directions. The set $\left\{W_{\alpha}\right\}$ of all such coordinate planes for this coordinate system will be called an $n$-framing of the space. For each $\alpha$, define $\pi_{\alpha}: W_{\alpha} \rightarrow \mathbb{R}^{n-f}$ by $\pi_{\alpha}\left(x_{i_{1}}, \ldots, x_{i_{n-f}}, y_{j_{1}}, \ldots, y_{j_{f}}\right)=\left(x_{i_{1}}, \ldots, x_{i_{n-f}}\right)$.

For any $n$-plane $W$ perpendicular to the base, the $n$-dimensional volume of $S$ in the directions of $W, \mathcal{V}_{W}(S)$, is defined as the $\mathcal{L}^{n}$ measure of the image $\Pi_{W}(\operatorname{Supp}(S))$ with multiplicities coming from two sources; the multiplicity of the current $S$ itself and the number of points (counted without orientations) $N\left(\Pi_{W} \mid S, x\right)[11],[2]$, in the preimage of $x$. This number is the Crofton multiplicity of the underlying map of sets, as used in the definition of the integral geometric measure of a rectifiable set. $\mathcal{V}_{W}(S)$ is then defined by:

$$
\mathcal{V}_{W}(S):=\int_{\Pi_{W}(\operatorname{Supp}(S))}\left(\sum_{y \in \Pi_{W}^{-1}(x)} \theta(y)\right) N\left(\Pi_{W} \mid S, x\right) d \mathcal{L}^{n} x
$$

This integral is not, in general, the same as the mass of the push-forward current $\left(\Pi_{W}\right)_{\#}(S)$, due to the canceling effect of orientation changes in the latter current. 
FiguRE 3.2: $S\left\llcorner B\left(y_{0}, \varepsilon\right)\right.$

Definition. Denote by $\left(\Pi_{W}\right)_{C}(S)$ the image current with support $\Pi_{W}(\operatorname{Supp}(S))$ in $W$ with the multiplicities of the current $S$ and the number of points (counted without orientations) $N\left(\Pi_{W} \mid S, x\right)$, which will be called the Crofton push-forward of $S$ onto $W$. Clearly $\mathcal{M}\left(\left(\Pi_{W}\right)_{C}(S)\right)=\mathcal{V}_{W}(S)$.

Given a positive number $\varepsilon$, the volume of $S\left\llcorner\pi^{-1}(B(x, \varepsilon))\right.$ satisfies:

$$
\begin{aligned}
\sqrt{1 / \gamma(n, m)} \sum_{\alpha} \mathcal{V}_{\alpha}\left(S\left\llcorner\pi^{-1}(B(x, \varepsilon))\right)\right. & \leq \mathcal{V}\left(S\left\llcorner\pi^{-1}(B(x, \varepsilon))\right)\right. \\
& \leq \sum_{\alpha} \mathcal{V}_{\alpha}\left(S\left\llcorner\pi^{-1}(B(x, \varepsilon))\right),\right.
\end{aligned}
$$

where $\Pi_{\alpha}: B(x, \varepsilon) \times \mathbb{R}^{m} \rightarrow W_{\alpha}$ is the orthogonal projection onto each of the $n$ planes of the $n$-framing, $\mathcal{V}_{\alpha}:=\mathcal{V}_{W_{\alpha}}$ as above, and $\gamma(n, m)=1+n m+\left(\begin{array}{c}n \\ 2\end{array}\right)\left(\begin{array}{c}m \\ 2\end{array}\right)+$ $\cdots+(\underset{\min (n, m)}{n})(\underset{\min (n, m)}{m})$ is the number of distinct $n$-planes, thus the number of distinct terms in the sum. This follows from the definition of the volume integral and the triangle inequality.

Definition. Let $y_{0} \in S$. For each $\varepsilon 0$, consider $S\left\llcorner B\left(y_{0}, \varepsilon\right)\right.$. Set $B_{\varepsilon}:=$ $\pi\left(\operatorname{Supp}\left(S\left\llcorner B\left(y_{0}, \varepsilon\right)\right)\right)\right.$ Define a comparison surface $F_{\varepsilon}$ in $B\left(y_{0}, \varepsilon\right)$, called a fence over $B_{\varepsilon}$ by, for $y_{0}=\left(x_{0}, z_{0}\right) \in B_{r} \times F$ :

$$
\operatorname{Supp}\left(F_{\varepsilon}\right) \cap \pi^{-1}(x)= \begin{cases}\left(x, z_{0}\right), & \text { if } x \in \operatorname{Int}\left(\pi\left(\operatorname{Supp}\left(S\left\llcorner B\left(y_{0}, \varepsilon\right)\right)\right)\right),\right. \\ C\left(\left(x, z_{0}\right), \varepsilon\right), & \text { if } x \in \partial\left(\pi\left(\operatorname{Supp}\left(S\left\llcorner B\left(y_{0}, \varepsilon\right)\right)\right)\right),\right.\end{cases}
$$


Figure 3.3: The "fence" $F_{\varepsilon}$

where $C\left(\left(x, z_{0}\right), \varepsilon\right)$ is the cone with vertex $\left(x, z_{0}\right)$ over $\operatorname{Supp}\left(\partial S\left\llcorner B\left(y_{0}, \varepsilon\right)\right) \cap\right.$ $\pi^{-1}(x)$.

This set is rectifiable, which follows from the rectifiability of the cone $C(\partial S)$ over $\partial S\left\llcorner B\left(y_{0}, \varepsilon\right)\right.$ with cone point $y_{0}$. The continuation of that cone, $2 C(\partial S)$, to radius $2 \varepsilon$ is also rectifiable, and the collar $C O(\partial S):=2 C(\partial S)-C(\partial S)$ is then also rectifiable. If $p \in C O(\partial S)$, map $p$ to $F_{\varepsilon}$ by:

$$
p=t\left(y-y_{0}\right) \mapsto\left(x, z_{0}+(t-1)\left(z-z_{0}\right)\right),
$$

where $y \in \operatorname{Supp}\left(\partial S\left\llcorner B\left(y_{0}, \varepsilon\right)\right), y=(x, z), x \in \partial B_{\varepsilon}\right.$, and $t \in[1,2]$. This is of course Euclidean notation; in general, these straight lines are replaced by geodesics. Since this mapping is Lipshitz (and in fact is a contraction map), the image is also rectifiable. Since the base $B_{\varepsilon}$ (the yard that is fenced in) is clearly rectifiable, $F_{\varepsilon}$ is rectifiable.

The multiplicity $\theta$ of the current on these cones is, on each ray, that of $S$ at the end of the ray, which is finite for almost-every ray for almost-all $\varepsilon$. Over a regular point of $S$ in $B_{\varepsilon}$, the cone will simply be a geodesic segment (for $\varepsilon$ sufficiently small we can assume that all pairs of points in $F$ of distance $\leq \varepsilon$ have a unique length-minimizing geodesic); over a pole point $x$ the cone might be significantly larger. $F_{\varepsilon}$ clearly is a rectifiable current, and in fact is a rectifiable section.

Set $s(\varepsilon):=\mathcal{V}\left(S\left\llcorner B\left(y_{0}, \varepsilon\right)\right)\right.$, and set $f(\varepsilon):=\mathcal{V}\left(F_{\varepsilon}\right)$. Since both $S\left\llcorner B\left(y_{0}, \varepsilon\right)\right.$ and $F_{\varepsilon}$ are rectifiable currents, and $S$ is by hypothesis minimizing, we have $s(\varepsilon) \leq f(\varepsilon)$. 
The remainder of the argument in [11], used to show the monotonicity formula, cannot be applied here directly. This is due to the inhomogeneous variation of the volume of the vertical part (the "fence") depending upon the dimension and magnitude of the vertical directions involved. However, that inhomogeneity can be separated out by the projections onto an $n$-framing as constructed above.

Let $\left\{W_{\alpha}\right\}$ be an $n$-framing of the space, and note that $s(\varepsilon) \leq f(\varepsilon) \leq$ $\sum_{\alpha} \mathcal{V}_{\alpha}\left(F_{\varepsilon}\right)$, by the definitions above. $\mathcal{H}^{n}\left(B_{\varepsilon}\right)$ is one of the terms of that sum, corresponding to $\alpha=(0,0)$. Set $f_{\alpha}(\varepsilon):=\mathcal{V}_{\alpha}\left(F_{\varepsilon}\right)$. For each $\alpha \neq(0,0)$, with say $k$ vertical directions,

$$
f_{\alpha}(\varepsilon)=\int_{\Pi_{\alpha}\left(B_{\varepsilon}\right)}\left(\int_{y \in C_{\alpha}\left(\left(x, z_{0}\right), \varepsilon\right)} N\left(\Pi_{\alpha} \mid F_{\varepsilon}, y\right) \theta(y) d \mathcal{L}^{k} y\right) d \mathcal{L}^{n-k} x
$$

by the general area-coarea formula, where $C_{\alpha}\left(\left(x, z_{0}\right), \varepsilon\right):=\Pi_{\alpha}\left(\operatorname{Supp}\left(F_{\varepsilon}\right)\right) \cap$ $\pi_{\alpha}^{-1}(x)$ is the cone over $\left(x, z_{0}\right)$ of the image $\left(\Pi_{\alpha}\right)_{\#}\left(\partial F_{\varepsilon}\right)$ in the fiber $\pi_{\alpha}^{-1}(x)$. For a generic $n$-framing $\Pi_{\alpha}$ will be almost-everywhere injective on $\partial F_{\varepsilon}$, in the Hausdorff $(n-1)$-dimensional measure on $\partial F_{\varepsilon}$, so that the Crofton multiplicity $N\left(\Pi_{\alpha} \mid \partial F_{\varepsilon}, x\right)$ will be almost-everywhere 1 on that boundary. $C_{\alpha}\left(\left(x, z_{0}\right), \varepsilon\right)$ is the image of all of the "fence posts" over points in $\Pi_{\alpha}^{-1}(x) \subset B_{\varepsilon}$, with multiplicities counted appropriately. Here $x=\Pi_{\alpha}\left(x, z_{0}\right) \in W_{\alpha}$ is identified with the image $\pi_{\alpha}\left(\Pi_{\alpha}\left(x, z_{0}\right)\right) \in \mathbb{R}^{n-k}$. Similarly, for $\delta<\varepsilon$ set $\left.C_{\alpha}\left(\left(x, z_{0}\right), \varepsilon\right)\right|_{\delta}:=$ $\Pi_{\alpha}\left(\operatorname{Supp}\left(F_{\varepsilon}\llcorner B(y, \delta))\right) \cap \pi_{\alpha}^{-1}(x) .\left.C_{\alpha}\left(\left(x, z_{0}\right), \varepsilon\right)\right|_{\delta}\right.$ is a subcone of $C_{\alpha}\left(\left(x, z_{0}\right), \varepsilon\right)$ corresponding to shortening the rays so as to remain within the smaller radius.

The proof of Theorem 3.1 will be completed after we establish two technical lemmas.

Lemma 3.2. There is an $A>0$ independent of $\varepsilon$ so that $f_{\alpha}(\varepsilon) \leq A \varepsilon^{n}$.

Proof. Denote by $\bar{\partial} C_{\alpha}$ the boundary $\left(\Pi_{\alpha}\right)_{\#}\left(\partial F_{\varepsilon}\right)=\partial\left(\Pi_{\alpha}\right)_{\#}\left(F_{\varepsilon}\right)$, not the boundary of the "enlarged" current $C_{\alpha}$ including added boundary components which appear when the Crofton multiplicity of the projection is included in the multiplicity of the projected current whose support is $\Pi_{\alpha}\left(\operatorname{Supp}\left(F_{\varepsilon}\right)\right)$. Note that $\bar{\partial} C_{\alpha}$ is the boundary of a rectifiable current $\left(\left(\Pi_{\alpha}\right)_{\#}\left(F_{\varepsilon}\right)\right.$ is rectifiable since it is an $n$-dimensional current in $\left.\mathbb{R}^{n}\right)$, thus for almost all $\delta$ the truncated cone $\left.C_{\alpha}\left(\left(x, z_{0}\right), \varepsilon\right)\right|_{\delta}$ will have rectifiable boundary by slicing.

As in [11], the volume of that portion of the cone which is the cone over a small piece $\Delta \bar{\partial} C_{\alpha}$ of the boundary is $(r / k) \mathcal{V}^{k-1}\left(\Delta \bar{\partial} C_{\alpha}\right)$, where $r<\varepsilon$ is the radius of that portion of the cone. Since the region is a cone, if the original piece of the boundary $\Delta \bar{\partial} C_{\alpha}$ is small enough, then the cone over that region will have Crofton multiplicity 1, unless that region is not transverse to the radial 
Figure 3.4: $C_{\alpha} \subset \mathbb{R}^{n}$, with multiplicities, and $\bar{\partial} C_{\alpha}$

direction at some point. If there is a set $T$ in $\bar{\partial} C_{\alpha}$ of positive $\mathcal{H}^{(n-1)}$-measure not transverse to the radial direction, then the mass of the cone over $\bar{T}$ will be 0 .

The infinitesimal change of radius $d \varepsilon$ on $S$ is projected by $\Pi_{\alpha}$ to a change in the radial direction of magnitude $(r / \varepsilon) d \varepsilon$. Set $f_{\alpha}(\varepsilon, x)$ to be the volume of the slice $C_{\alpha}\left(\left(x, z_{0}\right), \varepsilon\right)$ of by $\pi_{\alpha}$, where $\pi_{\alpha}$ is the projection onto the base $\mathbb{R}^{n-k}$ in $W_{\alpha}$. Then,

$$
\begin{aligned}
f_{\alpha}(\varepsilon, x) & =\int_{\Pi_{\alpha}\left(\operatorname{Supp}\left(\partial F_{\varepsilon}\right)\right) \cap \pi_{\alpha}^{-1}(x)}\left(\frac{r}{k}\right) d\left\|\left(\Pi_{\alpha}\right)_{\#}\left(\partial F_{\varepsilon}\right)\right\| \\
& \leq\left(\frac{\varepsilon}{k}\right) \frac{\partial}{\partial \varepsilon}\left(f_{\alpha}(\varepsilon, x)\right) .
\end{aligned}
$$

Thus, the slice mass-ratio $f_{\alpha}(\varepsilon, x) / \varepsilon^{k}$ is an increasing function of $\varepsilon$, wherever it is defined.

After establishing rectifiability of the currents under consideration, we will use a homotopy argument to relate the sizes of the various $f_{\alpha}(\varepsilon, x)$ to one another. In fact, we will show that $f_{\alpha}(\varepsilon, x)$ is a continuous function of $x$.

For $\mathcal{L}^{1}$-almost-every $\delta$ the slices $\left.\partial C_{\alpha}\left(\left(x, z_{0}\right), \varepsilon\right)\right|_{\delta} \subset C_{\alpha}\left(\left(x, z_{0}\right), \varepsilon\right)$ are rectifiable, being slices of a rectifiable current. This is the boundary of the cone $C_{\alpha}\left(\left(x, z_{0}\right), \varepsilon\right)$, truncated at $\delta$. For $\mathcal{L}^{1}$-almost-every $\delta$ this boundary is rectifiable. Since the region is a cone, this implies that the truncation at $\delta$ is rectifiable for all $\delta$. As before, we have that $\left.f_{\alpha}(\varepsilon, x)\right|_{\delta} \leq(\delta / k) \partial / \partial \delta\left(\left.f_{\alpha}(\varepsilon, x)\right|_{\delta}\right)$, which show first of all that $\left(\left.f_{\alpha}(\varepsilon, x)\right|_{\delta}\right) / \delta^{k}$ is an increasing function of $\delta$ as well. But this region is the 
Figure 3.5: $C_{\alpha}\left(\left(x, z_{0}\right), \varepsilon\right)$, with volume $f_{\alpha}(\varepsilon, x)$

image of a cone, with multiplicities counted, so that $\left(\left.f_{\alpha}(\varepsilon, x)\right|_{\delta}\right) / \delta^{k}$ is constant. Since the derivative $\partial / \partial \delta\left(\left.f_{\alpha}(\varepsilon, x)\right|_{\delta}\right)$ will be finite for $\mathcal{L}^{1}$-almost-all $\delta$, by rectifiability of the boundary, necessarily $f_{\alpha}(\varepsilon, x)$ will be finite for all $x$. Also, since homothetic expansion by $\delta_{1} / \delta_{2}$ in this slice space $\mathbb{R}^{k}$ takes $\left.\partial C_{\alpha}\left(\left(x, z_{0}\right), \varepsilon\right)\right|_{\delta_{1}}$ to $\left.\partial C_{\alpha}\left(\left(x, z_{0}\right), \varepsilon\right)\right|_{\delta_{2}}$, each boundary, in particular $\partial C_{\alpha}\left(\left(x, z_{0}\right), \varepsilon\right)$ itself, is rectifiable for each $x$.

Consider a small piece, $\Delta \partial C_{\alpha}\left(\left(x, z_{0}\right), \varepsilon\right)$, as before, of $\partial C_{\alpha}\left(\left(x, z_{0}\right), \varepsilon\right)$, so that the radial rays are transverse to $\partial C_{\alpha}\left(\left(x, z_{0}\right), \varepsilon\right)$ on $\Delta \partial C_{\alpha}\left(\left(x, z_{0}\right), \varepsilon\right)$. Restrict to a smaller ball centered at a point $p$ of $\Delta \partial C_{\alpha}\left(\left(x, z_{0}\right), \varepsilon\right)$, which defines

$$
Q(x):=\partial C_{\alpha}\left(\left(x, z_{0}\right), \varepsilon\right)\llcorner B(p, \delta) .
$$

For generic radii $\delta$ we can assume that this smaller piece $Q(x)$ has rectifiable boundary $\partial Q(x)$ in $\partial C_{\alpha}\left(\left(x, z_{0}\right), \varepsilon\right)$. Choose a positive $s<\varepsilon$ so that the radial distances $r$ all satisfy $s<r$, which is possible for sufficiently small $\delta$. Also, the cone over the boundary $C(\partial Q(x))$ with vertex $\left(x, z_{0}\right)$, inside of $\mathbb{R}^{k}$, is a $(k-1)$-dimensional cone in the $(k-1)$-dimensional cone over all of $\partial B(p, \delta) \cap \pi_{\alpha}^{-1}(x)$. Analogously to the fact that any compact $(k-1)$-dimensional subset of $\mathbb{R}^{k-1}$ is rectifiable, so is any $(k-1)$-dimensional subset of this cone. Thus the cone over $\partial Q(x)$ is rectifiable, and, being a cone, has mass bounded by $(s /(k-1)) \mathcal{M}(\partial Q(x)) \leq \mathcal{M}(C(\partial Q(x))) \leq(\varepsilon /(k-1)) \mathcal{M}(\partial Q(x))$. The constancy theorem applies, showing that the multiplicity is constant on that cone. This finally implies that $\partial Q(x)$ is indeed rectifiable for all radii. 
The cone over $Q(x), C(Q(x))$, has boundary $Q(x)$ plus the cone $C(\partial Q(x))$ over $\partial Q(x)$. The mass of $Q(x)$ is related to that of $C(Q(x))$ by

$$
\left(\frac{s}{k}\right) \mathcal{M}(Q(x)) \leq \mathcal{M}(C(Q(x))) \leq\left(\frac{\varepsilon}{k}\right) \mathcal{M}(Q(x)),
$$

similarly to the above.

Next, if $\left\{x, x_{1}\right\} \in B_{\varepsilon}$, then over any simple, smooth, unit-speed curve $\gamma$ from $\Pi_{\alpha}(x)$ to $\Pi_{\alpha}\left(x_{1}\right)$, construct the homotopy $C_{\alpha}(h):=\bigcup_{t \leq h} C_{\alpha}\left(\left(\gamma(t), z_{0}\right), \varepsilon\right)$ of currents. Over $\gamma$ the restriction of $C_{\alpha}(h)$ is a $(k+1)$-dimensional current in the $\mathbb{R}^{k+1}$ formed by the product of the fiber $\mathbb{R}^{k}$ and the curve, so that the homotopy $C_{\alpha}(h)$ is rectifiable.

Now, restrict the homotopy $C_{\alpha}(h)$ to the union of cones over coordinate slices of $B(p, \delta)$. The homotopy $C_{\alpha}(h)\left\llcorner C(B(p, \delta))\right.$ consists of $\bigcup_{t \leq h} C(Q(\gamma(t)))$, and its boundary consists of the $\bigcup_{t \leq h} Q(\gamma(t))$, together with $\bigcup_{t \leq h} C(\partial Q(\gamma(t)))$, and the two faces $C(Q(x))$ and $C(Q(\gamma(h)))$.

FiguRE 3.6

$C_{\alpha}(h)$. The top is $\bigcup_{t \leq h} Q(\gamma(t))$, and the sides are $\bigcup_{t \leq h} C(\partial Q(\gamma(t)))$

The mass of $C_{\alpha}(h)\llcorner C(B(p, \delta))$ shrinks to 0 as $h \rightarrow 0$, since it is a compact $(k+1)$-dimensional current in the $(k+1)$-dimensional Euclidean space $\pi_{\alpha}^{-1} \times$ $\gamma([0, h])$ (in fact, in a ball of radius $\varepsilon$ in $\mathbb{R}^{k}$ crossed with $\left.\gamma([0, h])\right)$. As $h \rightarrow 0$, the mass of this cylinder shrinks to 0 , so that the mass of the current must also (again, since this current is a $k+1$ dimensional current in a $k+1$-dimensional manifold, 
the constancy theorem implies that the multiplicity is constant). Similarly, the mass of $\bigcup_{t \leq h} C(\partial Q(\gamma(t)))$ shrinks to 0 as $h \rightarrow 0$.

The mass of $\bigcup_{t \leq h} Q(\gamma(t))$ can be evaluated as an iterated integral. Set $r((q, t))$ to be the radial distance from $\left(\gamma(t), z_{0}\right)$ to a point $(q, t) \in Q(\gamma(t))$. Since $\Pi_{\alpha}$ is almost-everywhere injective on $F_{\varepsilon}$ by assumption, there is almosteverywhere a lift $\bar{\gamma}(t)$ on $\partial B_{\varepsilon}$ so that at each $t, \bar{\gamma}(t)$ is the base of a fencepost whose top is the preimage of $(q, t)$. Since the length of the fenceposts are determined by the distance $u$ from $y_{0}$ to the base, $r=\sqrt{\varepsilon^{2}-u^{2}}$, the rate of change of $r$ is controlled,

$$
\left|\varlimsup_{v \rightarrow 0} \frac{r(q, \gamma(t+v))-r(q, \gamma(t))}{v}\right| \leq 2\left(\frac{\sqrt{\varepsilon^{2}-r^{2}}}{r}\right)\left|\frac{d \gamma}{d t}\right| \leq 2 \frac{\varepsilon}{s} .
$$

Then, using the area-coarea formula and this estimate of the change in radius as $t$ changes,

$$
\begin{aligned}
\mathcal{M}\left(\bigcup_{t \leq h} Q(\gamma(t))\right) & \leq \int_{0}^{h} \int_{Q(\gamma(t))} \sqrt{1+(2 \varepsilon / s)^{2}} d\|Q(\gamma(t))\| u d t \\
& =\int_{0}^{h} \sqrt{1+\left(\frac{2 \varepsilon}{s}\right)^{2}} \mathcal{M}(Q(\gamma(t)) d t \\
& \leq \int_{0}^{h} \sqrt{1+\left(\frac{2 \varepsilon}{s}\right)^{2}}\left(\frac{k}{s}\right) \mathcal{M}(C(Q(\gamma(t))) d t \\
& \leq \sqrt{1+\left(\frac{2 \varepsilon}{s}\right)^{2}}\left(\frac{k}{s}\right) \mathcal{M}\left(C_{\alpha}(h)\llcorner C(B(p, \delta)))\right.
\end{aligned}
$$

which shrinks to 0 as $h \rightarrow 0$.

Each $C(Q(\gamma(t)))$ is minimizing, since it is a $k$-dimensional current in an $\mathbb{R}^{k}$ (the fiber in $W_{\alpha}$ over $\gamma(t)$, which is a $k$-dimensional affine subspace of $W_{\alpha}$ ), so that

$$
\begin{aligned}
\mathcal{M}(C(Q(\gamma(h)))) \leq \mathcal{M}( & C(Q(x))) \\
& +\mathcal{M}\left(\bigcup_{t \leq h} Q(\gamma(t))\right)+\mathcal{M}\left(\bigcup_{t \leq h} C(\partial Q(\gamma(t)))\right),
\end{aligned}
$$

since the boundary of $C(Q(\gamma(h)))$ is the sum of the boundaries of $C(Q(x))$, $\bigcup_{t \leq h} Q(\gamma(t))$, and $\bigcup_{t \leq h} C(\partial Q(\gamma(t)))$. Conversely,

$$
\begin{aligned}
\mathcal{M}(C(Q(x))) \leq \mathcal{M}( & C(Q(\gamma(h)))) \\
& +\mathcal{M}\left(\bigcup_{t \leq h} Q(\gamma(t))\right)+\mathcal{M}(t \leq h C(\partial Q(\gamma(t)))),
\end{aligned}
$$


so that $\lim _{h \rightarrow 0} \mathcal{M}(C(Q(\gamma(h))))=\mathcal{M}(C(Q(x)))$. Since this is true everywhere on the cone, it implies continuity of $f_{\alpha}(\varepsilon, x)$.

Given $\varepsilon_{0}>0$, on the compact set $\overline{B_{\varepsilon_{0}}}$ there is a supremum $A \varepsilon_{0}^{k}$ for $f_{\alpha}\left(\varepsilon_{0}, x\right)$. Since the mass of the projected fence is given by:

$$
f_{\alpha}(\varepsilon)=\int_{\Pi_{\alpha}\left(B_{\varepsilon}\right)} f_{\alpha}(\varepsilon, x) d \mathcal{L}^{n-k}\left\llcorner\Pi_{\alpha}\left(B_{\varepsilon}\right),\right.
$$

this implies that

$$
f_{\alpha}(\varepsilon) \leq A \mathcal{L}^{n-k}\left(\Pi_{\alpha}\left(B_{\varepsilon}\right)\right) \varepsilon^{k} .
$$

Since $\mathcal{L}^{n-k}\left(\Pi_{\alpha}\left(B_{\varepsilon}\right)\right) \leq \omega_{n-k} \varepsilon^{n-k}$, this completes the proof of Lemma (3.2).

Lemma 3.3. For any $y_{0} \in S$, there is a constant $A>0$ so that $\mathcal{V}(S\llcorner$ $\left.B\left(y_{0}, \varepsilon\right)\right)<A \varepsilon^{n}$, thus the upper mass-density $\Theta^{*}\left(S, y_{0}\right)$ is finite at each point.

Proof. Since, for each $\alpha$, there is an $A=A_{\alpha}$ for which $f_{\alpha}(\varepsilon)<A_{\alpha} \varepsilon^{n}$, and

$$
\sqrt{\frac{1}{\gamma(n, m)}} \sum_{\alpha} \mathcal{V}_{\alpha}\left(S\llcorner B(y, \varepsilon)) \leq \mathcal{V}\left(S\llcorner B(y, \varepsilon)) \leq \sum_{\alpha} \mathcal{V}_{\alpha}(S\llcorner B(y, \varepsilon)),\right.\right.
$$

this Lemma follows directly from the preceding one.

Completion of the Proof of Theorem 3.1. Given $x_{0} \in P$, and any neighborhood $V$ of $x_{0}$ in $P$, there is an open neighborhood $U \subset V$ so that, for almost-all $x \in U$, there is a neighborhood of $x$ in $P$ of some maximum Hausdorff dimension $n-k$ (codimension $k$ ). Choose coordinates as above, and consider all projections onto $n$-planes of an $n$-framing $\left\{W_{\alpha}\right\}$. For each $n$-plane $W_{\alpha}$, if $\ell$ is the number of vertical directions in $W_{\alpha}$, we have the following cases:

$\triangleright$ CASE 1: $\ell \geq k$. There is an $A_{1}$ so that $\Pi_{\alpha}\left(\operatorname{Supp}\left(\left(S\left\llcorner\pi^{-1}(B(x, \varepsilon))\right)\right)\right)\right.$ has mass (including both multiplicities from $S$ and Crofton multiplicities of the projection) less than $A_{1} \varepsilon^{n-k}$ by Lemma 3.2 and the fact that projection does not increase the mass. This follows because $\Pi_{\alpha} \operatorname{Supp}\left(\left(S \cap \pi^{-1}(B(x, \varepsilon))\right)\right)$ can be covered by $C_{1} / \varepsilon^{k}$ balls, centered on $\operatorname{Supp}\left(\left(S \cap \pi^{-1}(x)\right)\right)$, of radius $2 \varepsilon$, for some $C_{1}>0$. As noted in Lemma 3.2, the upper mass-ratios on each of these balls is finite, so that on the compact set $\Pi_{\alpha}\left(\operatorname{Supp}\left(\left(S \cap \pi^{-1}(x)\right)\right)\right)$ there is an overall bound, $B_{1}$.

The total mass of $\Pi_{\alpha}\left(\operatorname{Supp}\left(S\left\llcorner\pi^{-1}(B(x, \varepsilon))\right)\right)\right.$ then is bounded by some $E_{1} \varepsilon^{n-k} \leq E_{1} \varepsilon^{n-\ell}$. 
$\triangleright$ CASE 2: $\ell<k$. In this case, the dimension of the projection of the pole over $x$ is of course bounded by $\ell$, and the densities are bounded as before, so that there is a cover of $\Pi_{\alpha}\left(\operatorname{Supp}\left(\left(S\left\llcorner\pi^{-1}(B(x, \varepsilon))\right)\right)\right)\right.$ by $C_{2} / \varepsilon^{k}$ balls of radius $2 \varepsilon$, for some $C_{2}>0$. Also, the upper mass-ratios on each of these balls is again finite, so that on the compact set $\Pi_{\alpha} \operatorname{Supp}\left(\left(S \cap \pi^{-1}(x)\right)\right)$ there is an overall bound, $B_{2}$.

The total mass of $\Pi_{\alpha}\left(\operatorname{Supp}\left(S\left\llcorner\pi^{-1}(B(x, \varepsilon))\right)\right)\right.$ then is bounded by some $C_{2} \varepsilon^{n-\ell}$.

These two cases imply that, for each $\alpha$, the projection of a cylindrical section $S\left\llcorner\pi^{-1}(B(x, \varepsilon))\right.$ onto $W_{\alpha}$ has mass bounded by $A_{\alpha} \varepsilon^{n-\ell}$, if $W_{\alpha}$ has $\ell$ vertical directions. Simple change of variables shows that the mass of the projection of the horizontally stretched image satisfies:

$$
\mathcal{V}_{\alpha}\left(\left(\nu_{1 / \varepsilon}\right)_{\#}\left(S\left\llcorner\pi^{-1}(B(x, \varepsilon))\right)\right) \leq A_{\alpha},\right.
$$

since only the $n-\ell$ horizontal directions are stretched, and those by a factor of $1 / \varepsilon$. However,

$$
\begin{aligned}
\mathcal{V}\left(\left(\nu_{1 / \varepsilon}\right)_{\#}(S)\left\llcorner\pi^{-1}(B(x, 1))\right)\right. & \leq \sum_{\alpha} \mathcal{V}_{\alpha}\left(\left(\nu_{1 / \varepsilon}\right)_{\#}(S)\left\llcorner\pi^{-1}(B(x, 1))\right)\right. \\
& \leq \sum_{\alpha} A_{\alpha}
\end{aligned}
$$

which is bounded, as required to satisfy the conditions of the Compactness Theorem.

Corollary 3.4. If $x$ is a pole point of $S$, with a pole $Z:=\pi^{-1}(x) \cap$ $\operatorname{Supp}(S)$ of Hausdorff dimension $k \in \mathbb{Z}$, with positive Hausdorff $k$-dimensional measure, then there is a sequence $\left\{\varepsilon_{i}\right\}$ with $\lim _{i \rightarrow \infty} \varepsilon_{i}=0$ and a constant $A>0$ for which $\mathcal{V}\left(S\left\llcorner\pi^{-1}\left(B\left(x, \varepsilon_{i}\right)\right)\right) \geq A \varepsilon_{i}^{n-k}\right.$.

Proof. For any $z \in Z$, since $z$ is not a boundary point of $S$ there is some $n$ framing $\left\{W_{\alpha}\right\}$ and some $\alpha$ for which the projection $\Pi_{\alpha}(z)$ is not a boundary point of the push-forward image $\left(\Pi_{\alpha}\right)_{\#}(S)$, because $\partial\left(\Pi_{\alpha}\right)_{\#}(S)=\left(\Pi_{\alpha}\right)_{\#}(\partial S)$ and general position. Thus, the mass-density of $\Pi_{\alpha}(z)$ in $\left(\Pi_{\alpha}\right)_{\#}(S)$ is at least one, so that the mass-density of $\Pi_{\alpha}(z)$ in the Crofton push-forward $\left(\Pi_{\alpha}\right)_{C}(\operatorname{Supp}(S))$ will be at least 1 as well. This implies that the lower mass-density $\Theta_{*}(S, z)$ is bounded below by $1 \leq \Theta_{*}(S, z)$, since the lower mass density of $\left(\Pi_{\alpha}\right)_{C}(\operatorname{Supp}(S))$ is at least 1 and $\mathcal{V}_{\alpha}(S) \leq \mathcal{V}(S)$. The lower mass-density of the $h$-cone $C_{h}$ satisfies this same bound, since the mass-density of the Crofton push-forward is still bounded below by 1 after a horizontal stretch. 
Take a sequence $\left\{\varepsilon_{i}\right\}$ with $\varepsilon_{i} \rightarrow 0$ for which

$$
\left\{\left(\nu_{1 / \varepsilon_{i}}\right)_{\#}\left(S\left\llcorner\pi^{-1}\left(B\left(x, \varepsilon_{i}\right)\right)\right)\right\}\right.
$$

converges to an $h$-cone $C_{h}$ of $S$ over $x$. Find an $\alpha$ for which $W_{\alpha}$ has $k$ vertical directions which maximizes $\mathcal{V}_{\alpha}\left(C_{h}\left\llcorner\pi^{-1}(B(x, 1))\right)\right.$. Then, using the lower massdensity bound above and a simple covering argument for the first inequality,

$$
\begin{aligned}
\frac{\omega_{n}}{\omega_{k}} \mathcal{H}^{k}(Z) & \leq \mathcal{V}\left(C_{h}\left\llcorner\pi^{-1}(B(x, 1))\right)\right. \\
& \leq \gamma(n, m) \mathcal{V}_{\alpha}\left(C_{h}\left\llcorner\pi^{-1}(B(x, 1))\right)\right. \\
& =\lim _{i \rightarrow \infty} \gamma(n, m) \mathcal{V}_{\alpha}\left(\left(\nu_{1 / \varepsilon_{i}}\right)_{\#}\left(S\left\llcorner\pi^{-1}\left(B\left(x, \varepsilon_{i}\right)\right)\right)\right)\right. \\
& =\lim _{i \rightarrow \infty} \gamma(n, m) \mathcal{V}_{\alpha}\left(S\left\llcorner\pi^{-1}\left(B\left(x, \varepsilon_{i}\right)\right)\right) / \varepsilon_{i}^{n-k} .\right.
\end{aligned}
$$

Then, for sufficiently large $i$,

$$
\mathcal{V}\left(S\left\llcorner\pi^{-1}\left(B\left(x, \varepsilon_{i}\right)\right)\right) \geq \frac{\omega_{n}}{\omega_{k} \gamma(n, m)} \mathcal{H}^{k}(Z) \varepsilon_{i}^{n-k} .\right.
$$

It should be noted that these $h$-cones may comprise two rather different types of phenomena. There may well be portions of $S$ of positive $S$-measure lying completely over the set of pole points, as well as radial portions tangent to horizontal rays emanating from the fibers of pole elements. It may also be that either of these pieces may have boundary over the set of pole points. Of course, if one has boundary, so does the other, so that the whole current $S$ will not have any interior boundary. It might also be (as given in the following example) that neither piece has interior boundary. The following example, showing that these poles may exist as volume-minimizers, is due to Gary Lawlor.

Example 3.5. Let $S_{n}$ be the graph of $w=1 /(n z)$, as a map $\varphi: \rightarrow \mathbb{C} P^{1}$, $S_{n} \in \operatorname{Cart}\left(\mathbb{C} P^{1} \times \mathbb{C} P^{1}\right)$. Certainly each $S_{n}$ is (non-uniquely) volume-minimizing in its homology class, since each is a complex submanifold of $\mathbb{C} P^{1} \times \mathbb{C} P^{1}$. The limit

$$
S:=\lim _{n \rightarrow \infty} S_{n}
$$

exists because the Cartesian norm of each $S_{n}$ is bounded, and will consist of the horizontal line $\mathbb{C} P^{1} \times 0$ and the vertical line $0 \times \mathbb{C} P^{1}$, each with multiplicity 1 . Note that the image on the fiber is a stable, minimizing 2-dimensional current. In Theorem 4.8, this will be shown to give the largest possible codimension of pole points. 
FiguRE 3.7: Orthogonal lines in $\mathbb{C} P^{1} \times \mathbb{C} P^{1}$ as a limit of smooth graphs

4. Main Results, Dimension-Reduction. Given a point $0 \in M$ which is a pole point of a volume-minimizing section $S \in \operatorname{Cart}(B)$, where $B$ is a $F$ bundle over $M$.

Let $Z=\pi^{-1}(0) \cap \operatorname{Supp}(S)$ be the pole over 0 , and assume that $\operatorname{dim}_{\mathcal{H}}(Z)=$ $k$.

The next result, using the abstract dimensional-reduction argument of $\mathrm{Si}$ mon [15], gives in this setting a direct restriction on the set of points $x \in M$ over which a pole can occur.

Let $S$ be, as usual, a volume-minimizing Cartesian current on a fiber bundle $B$ over a compact manifold $M$. For $x \in M$, let $U:=B_{r}$ be a geodesic ball of radius $r$ centered at $x$, and identify $\pi^{-1}\left(B_{r}\right)$ with $B_{r} \times F$. By Theorem 3.1, for all pole points $x \in P \cap B_{r}$ there is an $h$-cone.

Following the program of $[13$, Appendix A], let

$$
\mathcal{F}:=\left\{u_{y, \lambda}(x):=u(y+\lambda x)_{\mid B_{r}} \mid y \in B_{r}\right\} \cup\left\{v_{y} \text { an } h \text {-cone over } y \mid y \in B_{r}\right\},
$$

where the volume-minimizing current $S$ is given as the graph $S=\operatorname{graph}(u)$ for almost-all $x \in B_{r}$, and, for any $y \in B_{r}, v_{y}$ is a $h$-cone of $S$ centered at $y$ determined by some sequence of stretches. Since the proof of Theorem 3.1 still applies, in particular, the statement of Lemma 3.2 still holds for each stretch, and for the $h$-cone. The rest of the argument of Theorem 3.1 can then be repeated, showing that each of the stretched graphs, and the $h$-cones $v_{y}$, have an $h$-cone over each point. 
Proposition 4.1. The set $\mathcal{F}$ satisfies the following conditions:

(1) (Closure): If $|y| \leq r(1-\lambda), 0<\lambda<1$ and if $\varphi \in \mathcal{F}$, then $\varphi_{y, \lambda} \in \mathcal{F}$.

(2) (Existence of homogeneous degree-zero tangents): If $|y|<r$, if $\left\{\lambda_{k}\right\} \downarrow 0$ and if $\varphi \in \mathcal{F}$, then there is a subsequence $\left\{\lambda_{k^{\prime}}\right\}$ and $\psi \in \mathcal{F}$ such that $\varphi_{y, \lambda_{k^{\prime}}} \rightarrow \psi$ and $\psi_{y, \lambda}=\psi$ for each $\lambda>0$.

(Singular set hypothesis): A point $x \in B_{r}$ is a singular point of $\varphi \in \mathcal{F}$ if $\pi^{-1}(x) \cap$ $\overline{\operatorname{graph}(\varphi)}$ is not a single point. The set $\operatorname{sing}(\varphi)$ of singular points of $\varphi$ satisfies:

(3) $\operatorname{sing}(\varphi)=0$ if $\varphi$ is constant,

(4) If $|y| \leq r(1-\lambda), 0<\lambda<1$, then $\operatorname{sing}\left(\varphi_{y, \lambda}\right)=\lambda^{-1}(\operatorname{sing}(\varphi)-y)$,

(5) If $\varphi, \varphi_{k} \in \mathcal{F}$ with $\varphi_{k} \rightarrow \varphi$, then for each $\varepsilon>0$ there is a $k(\varepsilon)$ so that $B_{r}(0) \cap \operatorname{sing}\left(\varphi_{k}\right) \subset\left\{x \in \mathbb{R}^{n} \mid \operatorname{dist}(\operatorname{sing}(\varphi), x)<\varepsilon\right\}, \forall k \geq k(\varepsilon)$.

Proof. Conditions (1) and (2) follow from the definition of $\mathcal{F}$ and the properties of $h$-cones, (3) is trivial and (4) is straightforward.

Condition (5) follows from the specific nature of the set $\mathcal{F}$. If $\varphi, \varphi_{k} \in \mathcal{F}$ with $\varphi_{k} \rightarrow \varphi$, then since there are $y_{k} \in B_{r}$ and $\lambda_{k} \in(0,1]$ so that $\varphi_{k}=u_{y_{k}, \lambda_{k}}$, there is a subsequence $\{k(m)\}$ and $y_{0} \in B_{r}$ for which $y_{k(m)} \rightarrow y_{0}$, and either a $\lambda_{0} \in(0,1]$ for which $\lambda_{k(m)} \rightarrow \lambda_{0}$, or $\lambda_{k(m)} \rightarrow 0$. In either case, the weak convergence is, by definition, convergence in $\operatorname{Cart}\left(B_{r} \times F\right)$, by the compactness theorem, Proposition 1.1, since the Cartesian norms are uniformly bounded in $\operatorname{Cart}\left(B_{r} \times\right.$ $F)$. The boundedness of the Cartesian norms (here equivalent to boundedness of the mass since the fibers are compact) is explicitly shown in the proof of Theorem 3.1 for the $h$-cone case, and follows from the bounds established in that theorem in the case of $\lambda>0$. The bounds imply that a subsequence necessarily converges in $\operatorname{Cart}\left(B_{r} \times F\right)$. However, condition (5) presumes that $\varphi_{k} \rightarrow \varphi$, and uniqueness of limits in $\operatorname{Cart}(B)$ implies convergence of the full sequence. The remainder of condition (5); and with it the proof of the proposition; is established from the following lemmas, which are of some independent interest.

Lemma 4.2. If $S$ is a volume-minimizing Cartesian section, there is a positive number $f$ so that, if the projection $\Pi_{2}(Z):=\Pi_{2}\left(\pi^{-1}(0) \cap S\right)$ onto $F$ is contained within a ball of radius $f$, then $Z$ consists of a single point, and the graph is continuous at that point. If $Z$ is not a single point, then $\operatorname{dim}_{\mathcal{H}}(Z) \geq 1$.

Proof. For a positive number $f$, assume there is a $v_{0} \in \Pi_{2}(Z)$ so that $\Pi_{2}(Z)$ is contained in the geodesic ball $B\left(v_{0}, f\right)$ of radius $f$ centered at $v_{0}$. As in the proof of Proposition 1.3, write the section $u$ for which (almost-everywhere) $S=\operatorname{graph}(u)$ in terms of the distance $\delta$ from $v_{0}$, that is: in terms of local 
Riemann polar coordinates on $F$, (the polar coordinate system derived from a Riemann normal coordinate system centered at $v_{0}$ ):

$$
u(x)=\varphi^{-1}\left(\cos (\delta(x)) v_{0}+\sin (\delta(x)) a(x)\right)
$$

for some map $a:\left(B_{r} \backslash Q\right) \rightarrow S^{j-1}$, where $\delta(x):=\operatorname{dist}\left(v_{0}, \Pi_{2}(x)\right)$ and $\varphi$ is a Riemann normal coordinate chart. This decomposes $u$ according to polar coordinates $y=(\delta, a)$ in the exponential image $u(x)=\operatorname{Exp}_{v_{0}}(y)=\varphi^{-1}(y)$, for $y \in T_{*}(F, v)$.

Writing the functional for the volume of the graph, $\mathcal{V}(\operatorname{graph}(u))$, in terms of $\delta$ as well, results in the following, since the radial directions are perpendicular to the spherical images corresponding to constant values of $\delta$.

$$
\mathcal{V}(\operatorname{graph}(u))=\int_{B_{r}} \sqrt{\sum_{\ell \geq 0}^{j-1} \sin ^{2 \ell}(\delta)\left\|(1+d \delta) \wedge(\nabla a)^{\wedge \ell}\right\|^{2}} d V
$$

For an $f>0$ to be determined, assume there is a $r>0$ sufficiently small so that $\delta<f$ on $B_{r}$, and replace $u$ by the linear contraction from the boundary values, given in polar coordinates by: $w(\rho, \theta)=\varphi^{-1}\left(\cos (\rho \delta(\theta)) v_{0}+\sin (\delta(\theta)) a(\theta)\right)$ where $\delta(\theta):=\delta(r \theta)$ and $a(\theta):=a(r \theta)$ are the boundary values of those maps. Then,

$$
\begin{aligned}
\mathcal{V}(\operatorname{graph}(w))=\int_{0}^{r} \int_{S^{n-1}}\left[\sum_{\ell=0}^{j-1} \sin ^{2 \ell}(\rho \delta(\theta)) \|\left(1+\delta d \rho+\frac{\rho d_{\theta} \delta}{\rho}\right)\right. \\
\left.\wedge\left(\frac{\nabla_{\theta} a}{\rho}\right)^{\wedge \ell} \|^{2}\right]^{(1 / 2)} \rho^{n-1} d V_{S^{n-1}} d \rho,
\end{aligned}
$$

where $d_{\theta}$ and $\nabla_{\theta}$ indicate derivatives as functions on the polar-coordinate $\theta \in$ $S^{n-1}$.

For a given $f<\pi / 2$, since $0<\delta(\theta)<f, \rho>\sin (\rho \delta(\theta)) / \sin (\delta(\theta))$ for all $\theta$ and for all $0<\rho<r<1$. Then

$$
\begin{aligned}
& V\left(S_{\mid B_{r}}\right) \leq V(\operatorname{graph}(w)) \\
& \leq \int_{0}^{r} \int_{S^{n-1}}\left[\sum_{\ell=0}^{j-1}(\rho \sin (\delta(\theta)))^{2 \ell}\left\|\left(1+\delta d \rho+d_{\theta} \delta\right) \wedge\left(\frac{\nabla_{\theta} a}{\rho}\right)^{\wedge \ell}\right\|^{2}\right] \rho^{n-1} d V_{S^{n-1}} d \rho \\
& \leq(1+f) \int_{0}^{r} \int_{S^{n-1}}\left[\sum_{\ell=0}^{j-1} \sin ^{2 \ell}(\delta(\theta))\left\|\left(1+\frac{d_{\theta} \delta}{r}\right) \wedge\left(\frac{\nabla_{\theta} a}{r}\right)^{\wedge \ell}\right\|^{2}\right]^{(1 / 2)} \rho^{n-1} d V_{S^{n-1}} d \rho \\
& \leq \frac{(1+f)}{n} r \frac{d}{d r}\left(V\left(S_{\mid B_{r}}\right)\right),
\end{aligned}
$$


and so, as earlier, $V\left(S_{\mid B_{r}}\right) \leq A r^{n /(1+f)}$.

However, if $f$ is sufficiently small, then $n /(1+f)>n-1$, so that, by Lemma 3.4, $\operatorname{dim}(Z)<1$. However, since $Z=\lim _{i \rightarrow \infty} u\left(B_{r_{i}}\right)$, where $r_{i} \downarrow 0$, is the limit of a nested sequence of closed, connected sets, it is closed and connected. Thus, if $Z$ consists of more than one point, it must be of dimension at least 1 , and such a number $f$ cannot be found.

Lemma 4.3. If $x \in M$ is a pole point of $S$, with a nontrivial pole $Z:=$ $\pi^{-1}(x) \cap \operatorname{Supp}(S)$, and if $\left\{\varepsilon_{i}\right\}$ is a sequence of stretches so that $\left(\nu_{1 / \varepsilon_{i}}\right)_{\#}(S\llcorner$ $\left.\pi^{-1}\left(B\left(x, \varepsilon_{i}\right)\right)\right) \rightarrow C_{h}(x)$, where $C_{h}(x)$ is the $h$-cone of $S$ at $x$, then $\mathcal{V}\left(S_{\mid B\left(x \varepsilon_{i}\right)}\right)$, the volume of that portion of the minimizing current lying over $B\left(x, \varepsilon_{i}\right)$, is at least $A \varepsilon_{i}^{n-1}$ for some $A>0$.

Remark. The main reason we have to restrict the radii to those coming from a sequence of stretches leading to an $h$-cone is to ensure that the mass-ratios converge.

Proof. From Lemma 4.2, necessarily the singularity must have dimension at least one. The rest of the statement follows from Corollary 3.4.

Completion of the proof of Proposition 4.2. The only remaining condition to verify is that, for a sequence $u_{y_{k}, \lambda_{k}} \rightarrow v_{y_{0}}$ (or, if $\lambda_{k} \not \rightarrow 0, u_{y_{k}, \lambda_{k}} \rightarrow$ $\left.u_{y_{0}, \lambda_{0}}\right), y_{k} \rightarrow y_{0}$, there is, for each $\varepsilon>0$, an $k(\varepsilon)>0$ so that $B_{r}(0) \cap$ $\operatorname{sing}\left(u_{y_{k}, \lambda_{k}}\right) \subset\left\{x \in \mathbb{R}^{n} \mid \operatorname{dist}\left(\operatorname{sing}\left(v_{y_{0}}\right), x\right)<\varepsilon\right\}, \forall k>k(\varepsilon)$. If not, there is an $\varepsilon>0$ for which there is a sequence $\{k(m)\}$ and $x_{m} \in \operatorname{sing}\left(u_{y_{k(m)}, \lambda_{k(m)}}\right) \cap B_{r}(0)$ with $\operatorname{dist}\left(x_{m}, \operatorname{sing}\left(v_{y}\right)\right)>\varepsilon$, and so that $x_{m} \rightarrow x_{0}$.

Now, Lemma 4.3 implies that

$$
\mathcal{V}\left(u_{y_{k(m)}, \lambda_{k(m)}} \mid B_{\lambda_{k(m)}}\left(y_{k(m)}\right)\right) \geq A\left(\lambda_{k(m)}\right)^{n-1}
$$

for some $A>0$ independent of $m$. However, the hypothesis that $x_{0}$ is not singular for $v_{y_{0}}$ implies that $\mathcal{V}\left(v_{y_{0} \mid B_{t}}\left(y_{0}\right)\right) \leq B t^{n}$. But, for any $t>0$, there is a $m \gg 0$ for which $B_{t / 2}\left(y_{k(m)}\right) \subset B_{t}\left(y_{0}\right)$. Since the Cartesian norms, hence the volumes, of the sequence converge to the volume of the limit, this is a contradiction.

Corollary 4.4. Let $P$ be the singular locus of pole points $x \in M$ of a volume-minimizing section $S \in \operatorname{Cart}(B)$. Then there is an integer $d \in[0, n-1]$ for which $\operatorname{dim}_{\mathcal{H}}(P) \leq d$, for which there also is a tangent $h$-cone $\psi$ of $S$ and a $d$-dimensional subspace $L \subset \mathbb{R}^{n}$ for which each point of $L \cap B_{1}(0)$ is a pole point of $\psi$, and $\psi$ is invariant under translations along $L$. 
Proof. Proposition 4.1 verified the conditions which imply these conclusions, from Simon's general dimension-reduction theorem [13, App. A].

As before, let $Z=\pi^{-1}(0) \cap \operatorname{Supp}(S)$ be the pole over $0 \in M$, and assume that $\operatorname{dim}_{\mathcal{H}}(Z)=k$. Define, for $u: B_{r_{0}}^{n} \rightarrow F$, and $1>\lambda>0$, the $\lambda$-stretched functional $\mathcal{V}_{\lambda}(u)$ by

$$
\mathcal{V}_{\lambda}(u):=\mathcal{V}\left(u_{1 / \lambda}\left\llcorner B_{r_{0} \lambda} \times F\right) / \lambda^{n-k},\right.
$$

where $u_{\gamma}:=u_{0, \gamma}$ is defined by $u_{\gamma}(x):=u(\gamma x)$. Note that, as currents,

$$
\operatorname{graph}\left(u_{\gamma}\right)\left\llcorner B_{r} \times F=\left(( \nu _ { 0 , 1 / \gamma } ) _ { \# } ( \operatorname { g r a p h } ( u ) ) \left\llcornerB_{r} \times F .\right.\right.\right.
$$

This can be extended to a functional $\mathcal{V}_{\lambda}$ on $\operatorname{Cart}\left(B_{r_{0}} \times F\right)$ by taking limits as usual, defining $\mathcal{V}_{\lambda}(T)$ to be $\mathcal{V}\left(\left(\nu_{\lambda}\right)_{\#}(T)\left\llcorner B_{r_{0} \lambda} \times F\right) / \lambda^{n-k}\right.$. Note that the stretched sections $u_{\gamma, 0}$ have appropriate boundary conditions, which are $\left(u_{\gamma}\right)_{\mid \partial B_{r_{0}}}(x)=u(\gamma \cdot x)$, for $x \in \partial B_{r_{0}}$. If $S\left\llcorner B_{r_{0}} \times F\right.$ minimizes volume, then clearly $\left(\nu_{1 / \lambda}\right)_{\#}(S)\left\llcorner B_{r_{0}} \times F\right.$ minimizes $\mathcal{V}_{\lambda}$ subject to the boundary conditions given as above.

The next results address the possible existence of $n$-dimensional pieces of the minimizer $S$ over the set of pole points $P$. Note that this condition only can hold if the dimension of the intersection with a generic fiber is the same as the codimension of the set $P$ in a neighborhood of the intersection. In this case, since the fibers of the map $\pi$ slice $S$ into $S$-almost everywhere rectifiable slices, almost-all of the intersections $\operatorname{Supp}(S) \cap \pi^{-1}(x)$, for $x$ in this neighborhood of $P$ over which lies a positive-measure piece of $S$, will be rectifiable. Thus, the dimension $k$ of $\operatorname{Supp}(S) \cap \pi^{-1}(x)$ is an integer. Also, slicing by codimension $k$ planes transverse to a regular point of a fiber $\operatorname{Supp}(S) \cap \pi^{-1}(x), \mathcal{H}^{(n-k)}\llcorner P$ almost every transverse slice is also rectifiable.

Proposition 4.5. If, for a neighborhood $U \subset P$ of $x \in P, S\left\llcorner\pi^{-1}(U)\right.$ has positive $n$-dimensional Hausdorff measure, then all fibers $S_{x}:=\operatorname{Supp}(S) \cap \pi^{-1}$ are stable minimal currents (perhaps with boundary) within the space of rectifiable $k$-dimensional currents in $\pi^{-1}(x)$.

Proof. This proof uses the dimension-reduction argument above, from Corollary 4.4. If such a neighborhood $U$ exists, then there is an $h$-cone $\psi$ which has poles exactly over a dimension- $n-k$ linear subspace $L \cap B_{r}^{n}(0)$, and so that $\psi$ is invariant under translations along $L$. Here the $d$ of Corollary 4.4 is $n-k$ by the dimension restrictions.

$\psi$ then defines a transversal $h$-cone $\varphi$, where $B_{r}^{k}(0):=B_{r}^{n}(0) \cap L^{\perp}$, by projection. 
Assume that the singular fiber $S_{0}$ of $\varphi$ over 0 (which is the singular fiber of $S$ over 0 ) is not a stable minimal current relative to its boundary. Then, since $\varphi\left\llcorner\pi^{-1}(L)\right.$ is the product of $L$ with $S_{0}, \partial \varphi\left\llcorner\pi^{-1}(L)=\partial S_{0}\right.$ as well, and a deformation of the singular fibers $S_{x}$, relative to $\partial S_{x}$, for $x \in L$, can be applied to lessen the volume of $\varphi L\left(\pi^{-1}(L) \cap B_{r} \times F\right)$ while preserving the boundary. Moreover, since $S_{\lambda_{i}}$, for some sequence $\lambda_{i} \downarrow 0$, converges in $\operatorname{Cart}\left(B_{r} \times F\right)$ to $\varphi$, there will be an $(n-k)$-dimensional neighborhood $L_{i}$ of 0 in the stretch $\nu_{i}(P)$ so that, for $x \in L$, and for $x_{i} \in L_{i}$ so that $x_{i} \rightarrow x$, the singular fibers $S_{i, x_{i}}$ will be converging to $S_{x}$. Thus, for $i$ sufficiently large, $S_{i, x_{i}}$ will also not be stable minimal, and so the process of shrinking the interiors of $S_{i, x_{i}}$ can be again applied to lessen the volume of $S_{i}\left\llcorner\pi^{-1}\left(L_{i}\right)\right.$. Since the contribution of $S_{i}\left\llcorner\left(\pi^{-1}\left(L_{i}\right) \cap B_{r} \times F\right)\right.$ to the stretched energy $\mathcal{V}_{\lambda_{i}}\left(S_{i}\left\llcorner\left(\pi^{-1}\left(L_{i}\right) \cap B_{r} \times F\right)\right)\right.$ is (due to the scale factor $\left.\lambda^{n-k}\right)$ bounded, and converges to $\mathcal{V}\left(\varphi\left\llcorner\left(\pi^{-1}(L) \cap\right.\right.\right.$ $\left.B_{r} \times F\right)$ ), that deformation will lessen the $\mathcal{V}_{\lambda_{i}}$-energy of $S_{i}$. However, $S_{i}$ is a minimizer of that energy, so the fiber $S_{0}$ must be a stable minimal current in $F$, relative to $\partial S_{x}$.

Remark. This argument can be slightly altered to show that $S_{x}$ must be minimizing with respect to images of all deformations (homotopies) maintaining the boundary conditions, rather than just for the small deformations implied by stability. Simply perform the deformations on the poles $S_{i, x_{i}}$ of $S_{i}$ as above to a lower-volume current than $S_{i, x_{i}}$ in a small annular region, with a large enough inner portion so that the increase in stretched volume in the deformation region is canceled by the interior with the smaller singular fibers.

Corollary 4.6. If $\mathcal{H}^{n}\left(S\left\llcorner\pi^{-1}(P)\right)>0\right.$, and if $\partial S_{x} \neq 0$, for some $x \in P$, then $\partial S_{x}$ is the image under $\left(\Pi_{2}\right)_{\#}$ of the slice of $\varphi$ over $\partial B^{n-k}(r, 0)$.

Proof. This follows from the conditions that the boundary of the fibers $S_{x}$ must coincide with the radial portions.

Proposition 4.7. $\operatorname{dim}(P)<n-1$. That is, the set of pole points has codimension greater than one.

Proof. Let $x \in P$ be a generic pole point of $S$. Let $\left\{\lambda_{i}\right\}$ be a sequence $\lambda_{i} \downarrow 0$ so that the stretches $S_{\lambda_{i}}:=S_{i}$ converge to $\psi \cdot \operatorname{dim}(P)$ cannot be greater than $n-1$, since the generic fiber must have dimension at least one, by Lemma 4.2.

If $\operatorname{dim}(P)=n-1$, then the conditions of Proposition 4.5 necessarily hold. Assume first that there is some boundary to $S_{x}$ for some $x$. Since $k=1$, almostevery pole point $x \in P$ will have a pole $S_{x}$ of dimension at most one.

An $h$-cone $\psi$ at a generic $x \in P$ for $S_{\mid U}$ would have an $(n-1)$-dimensional horizontal subspace $L$ as in Corollary 4.5, and would be invariant under translations along $L$. Thus, the vertical component of the transverse cones $\varphi$ would all 
be congruent, and each would meet with the radial portion orthogonally at the common boundary. That common boundary (within $\psi$ ) would be a product of the boundaries $\partial S_{x}$ (which are a pair of points) of each fiber $S_{x}$ with the linear subspace $L$, by the invariance under translation. Also, for the same reason the transverse cones $\varphi$ are all rectifiable. Since they are connected, minimizing, and of dimension at most one, each is necessarily a geodesic segment.

It is easy to construct a comparison graph $\zeta$ with the same boundary but less stretched volume than the $h$-cone $\psi$, by shaving off the corners of each of the transverse $h$-cones $\varphi$. In order to preserve the boundary conditions, the shaving must be stopped prior to the boundary. Specifically, for some sufficiently small $\delta$, a neighborhood $A_{\delta}$ of radius $\delta$ centered at a boundary point of $S_{x}$ is a pair of segments of length $\delta$ meeting orthogonally at the boundary point of $S_{x} . A_{\delta}$ can be replaced by the geodesic segment connecting the two boundary points $\partial A_{\delta}$. This construction can be repeated for both boundary points of $S_{x}$ for all $x \in P$ within $r / 2$ of the center 0 of a ball $B_{r}^{n}(0)$, for some $r>0$ so that $B_{r}^{n}(0) \cap P \subset U$. Then, triangles of area $\frac{1}{2} \delta^{2}$ will fill in the boundary at each point of $\partial B_{r / 2}^{n} \cap P$. The new graph $\zeta$ will have stretched volume

$$
\begin{aligned}
& \mathcal{V}_{\lambda_{i}}(\zeta)=\mathcal{V}_{\lambda_{i}}(\psi) \\
& -\left(\left(1+\lambda_{i}-\sqrt{1+\lambda_{i}^{2}}\right) \delta \omega_{n-1}\left(\frac{r \lambda_{i}}{2}\right)^{n-1}-\frac{1}{2} \delta^{2} \lambda_{i} \omega_{n-2}\left(\frac{r \lambda_{i}}{2}\right)^{n-2}\right) /\left(\lambda_{i}\right)^{n-1},
\end{aligned}
$$

for which there clearly will be a $\delta>0$ so that $\mathcal{V}_{\lambda_{i}}(\zeta)<\mathcal{V}_{\lambda_{i}}(\psi)$.

FiguRE 4.1: Shaving the corners of $\psi$ to construct $\zeta$ 
For sufficiently small $\lambda_{i}$ (sufficiently large stretches), the construction of $\zeta$ from $\psi$ can be repeated with $S_{\lambda}$ replacing $\psi$, generating a sequence $\zeta_{i}$ of comparison surfaces. Clearly $\mathcal{V}_{\lambda_{i}}\left(\zeta_{i}\right)<\mathcal{V}_{\lambda_{i}}\left(S_{i}\right)$ for sufficiently small $\lambda_{i}$. This contradiction shows that there can be no boundary to a generic $S_{x}$.

The remaining case is that a generic $S_{x}$ is a closed, length-minimizing geodesic. However, $\operatorname{Supp}\left(S_{x}\right)$ is the limit of the images $u_{m}(B(x, \varepsilon))$, where $\left\{u_{m}\right\}$ is a minimizing sequence of smooth graphs $u_{m}: B^{n}(r, 0) \rightarrow F$. Moreover, the transverse $h$-cones $\varphi$ are also Cartesian sections, and the $\operatorname{support} \operatorname{Supp}\left(S_{x}\right)$ is the limit of the images $v_{m}\left(B^{1}(x, \varepsilon)\right)=v_{m}(-\varepsilon, \varepsilon)$ as well, for $v_{m}$ defined by $v_{m}:=\left.\left(u_{m}\right)\right|_{\mid W_{x} \cap B^{n}(r, 0)}$, where $W_{x}$ is the affine 2-dimensional subspace meeting $L$ perpendicularly at $x \in L$. Thus the graphs of $v_{m}$ are a sequence of smooth graphs converging to $\varphi$. However, since $v_{m}(-\varepsilon, \varepsilon)$ is on the one hand a sequence approaching a closed curve (since $\partial S_{x}=0$ ) and is on the other hand, taking $\varepsilon \rightarrow 0$, homotopically trivial, that closed curve must be homotopically trivial. (This requires the slight extension of Proposition 4.5 discussed in the remark after the proof of that proposition.) This contradicts the fact that $S_{x}$ is massminimizing up to deformations, unless $S_{x}$ is a single point, completing the proof of the proposition.

The next result is a re-statement of our main theorem, Theorem 0.1 of the Introduction. Moreover, since its proof is essentially local, it also provides a proof of Theorem 0.2. Note for that result that the linear (or geodesic) constructions of the comparison surfaces such as the "fence" of Proposition 3.1 necessitate that the subsets of Euclidean spaces considered be convex for Theorem 0.2.

Theorem 4.8. The set of pole points $P$ of a volume-minimizing Cartesian section $S \in \operatorname{Cart}(B)$ over a compact Riemannian manifold $M$ has codimension at least 3 in $M$, unless there is a stable minimal 2-dimensional current $T \subset F$ (without boundary), in which case there may be a codimension 2 set of pole points with poles which are $\mathcal{H}^{(n-2)}\llcorner P$-almost-all stable, minimal, 2-dimensional currents without boundary.

Remark. Example 3.5 shows that the codimension 2 possibility is sharp, in that there are minimizing rectifiable sections with codimension 2 sets of pole points.

Proof. In a neighborhood of a pole point $x$, which we denote by 0 , the conditions of the construction preceding Proposition 4.1 hold, thus the conclusions of Corollary 4.4 also hold, so that there is an integer $d \geq p$, which is the maximum dimension of any neighborhood of $P$, and a $\mathcal{V}_{h}$-minimizing $h$-cone $\psi$ which has poles exactly over a dimension- $d$ linear subspace $L \cap B_{r}^{n}(0)$, and so that $\psi$ is invariant under translations along $L$. 
$\psi$ then defines a transversal $h$-cone $\varphi \in \operatorname{Cart}\left(B_{r}^{n-d}(0) \times F\right.$, where

$$
B_{r}^{n-d}(0):=B_{r}^{n}(0) \cap L^{\perp}
$$

by projection. From the decomposition above, the image $\left(\Pi_{2}\right)(\operatorname{Supp}(\psi))$ is the same as $\left(\Pi_{2}\right)(\operatorname{Supp}(\varphi))$; and

$$
Z=\Pi_{2}(\operatorname{Supp}(\varphi)) \supset \Pi_{2}(\operatorname{Supp}(\partial \varphi)) .
$$

The containment above will be strict only if (up to sets of measure 0) all (by invariance) of the fibers $S_{x}$ of $\psi$ are not contained in the images of the radial components of the transverse $h$-cones, in which case the dimension of $S_{x}$ would necessarily be the codimension of $P$ at $x$, and the conditions of Proposition 4.5 will hold, since otherwise the set $\bigcup_{\{x \in P\}} S_{x}$ would contain an open set in $S$ of dimension less than $n$. If the containment is strict, each fiber $S_{x}$ will be a stable, minimal, rectifiable current as shown in Proposition 4.5, whose boundary is the image of the radial part of the transverse $h$-cone.

In the case that $\Pi_{2}(\operatorname{Supp}(\varphi))=\Pi_{2}(\operatorname{Supp}(\partial \varphi))$, and in the previous case where $\Pi_{2}\left(\partial S_{x}\right)=\Pi_{2}(\operatorname{Supp}(\partial \varphi))$, since $\varphi$ is a rectifiable section, then $\Pi_{2}(\operatorname{Supp}(\partial \varphi))$ is the union of a countable number of Lipshitz images of the $(n-d-1)$-dimensional manifold $\partial\left(B_{r}^{(n-d)}\right)$.

Thus, if $\operatorname{dim}_{\mathcal{H}} Z=n-d$, then, $Z$ will be minimizing, and if $\operatorname{dim}_{\mathcal{H}} Z<n-d$, then $\operatorname{dim}_{\mathcal{H}} Z \leq n-d-1$, since it is rectifiable.

If the statement of the theorem were not true, then either $n-d=1$ or 2 . The case $n-d=1$ has been eliminated by Proposition 4.7. If $n-d=2$, the argument above implies that the dimension of each pole is 1 or 2 . If the dimension of the poles is 2 , they may have some one-dimensional boundary given in terms of the transverse $h$-cone $\varphi$ as $\left(\Pi_{2}\right)_{\#}(\partial \varphi)$. If the dimension of the pole is one, the poles themselves are $\left(\Pi_{2}\right)_{\#}(\partial \varphi)$, which defines a rectifiable closed curve whose trace is the pole $Z$.

The following lemma will complete the proof of the theorem in the case $n-d=2$, with dimension-2 poles. This case does occur as a minimizing graph, as shown by Example 3.5 above.

Lemma 4.9. Each pole $S_{x}$ is a 2-dimensional stable, minimal current in $F$ with $\partial S_{x}=0$.

Proof. As before, for some sequence $\lambda_{i} \downarrow 0$, the stretches $S_{i}:=S_{\lambda_{i}}$ will converge to the $h$-cone $\psi$. Moreover, $S_{i}$ minimizes the stretched volume $\mathcal{V}_{\lambda_{i}}$.

The only part of this statement not yet established is that $\partial S_{x}=0$. If there is a boundary to $S_{x}$, then an argument similar to (but slightly more involved 
than) that of Proposition 4.5 again constructs a comparison surface with less $\mathcal{V}_{\lambda_{i}}$-energy than the $\lambda_{i}$-stretch $S_{i}$ over a ball $B(0, r)$ in the base.

Since $\partial S_{x}$ is the image of $\left(\Pi_{2}\right)_{\#}(\partial \varphi)=S^{1}$, the "shaving" construction can be carried out more easily on only a part of $\partial S_{x}$ which maps in a 1-1 fashion from an $\operatorname{arc}$ of $S^{1}$. There, replace that portion of the transverse $h$-cone $\varphi$ within $\delta$ of the boundary arc with the surface generated by the geodesic segments joining points $\delta$ from the boundary arc. In the radial portion of $\varphi$, choose the point $\delta$ along the radial ray beginning at a boundary point $y$. In the current $S_{x}$, choose the point $\delta$ from $y$ along a geodesic joining $y$ to some point $z$ in $S_{x}$ which is far from the boundary relative to $\delta$, yet still close enough so that there is a unique geodesic joining them within $S_{x}$. Since $S_{x}$ is minimal, it is regular except for an isolated set of points, so for a sufficiently small arc of the boundary this construction will be well-defined, independently of the choice of (sufficiently small) $\delta$.

Replacing the two pieces of $\varphi$ on either side of $\partial S_{x}, S_{0}$, by this surface $\sigma(\delta, \ell)$ will lessen the $\mathcal{V}_{\lambda_{i}}$ energy by a factor $0<\gamma\left(\delta, \ell, \lambda_{i}\right)<1$, for $\gamma=\gamma\left(\delta, \ell, \lambda_{i}\right)$ perhaps slightly less than the $\left(1+\lambda_{i}-\sqrt{1+\lambda_{i}^{2}}\right) \delta \pi(r / 2)$ found in Proposition 4.5 (with $n=2$ ), but the shrinking will be proportional to $\delta$ and the length $\ell$ of the shaving. It is necessary to "cap off" this shaving at either end, by adding in a triangle at each end of energy bounded by a constant times $\delta^{2} \lambda_{i}$, so that, for sufficiently small $\delta$ the shaved area, including the caps, will still fit within the required bounds.

FIGURE 4.2: Shaving the boundary of $S_{x}$ 
Repeating this construction for all $x$ in a neighborhood with closure in the interior of $B(0, r)$ lessens the $\mathcal{V}_{\lambda_{i}}$ energy of the $h$-cone $\psi$ by a factor of at least $\gamma / 2$. It will finally be necessary to cap off the interface between the shavings and the remainder of $\psi$. The cap is the convex hull of $S_{0} \cup \sigma(\delta, \ell)$ over each point in the circle $\partial B^{2}(0, r)$. That is, the cap over each point is the 3 -dimensional current $G$ so that $\partial G=S_{0}-\sigma(\delta, \ell)+T_{1}+T_{2}$, where $T_{1}$ and $T_{2}$ are the triangles added to each shaved vertical slice. The added energy due to these caps will be asymptotically proportional to $\ell \delta^{2}$, and $\lambda_{i}$, so that for sufficiently small $\delta$ the altered current will have a factor of $\gamma / 3$ less energy.

As in Proposition 4.6, this entire shaving process can be repeated for $S_{i}$, which will then provide a surface with less $\mathcal{V}_{\lambda_{i}}$ energy than $S_{i}$, contradicting the construction of $S_{i}$. Since the assumption was that there was some boundary to $S_{x}$ in $\varphi$, the contradiction implies that $\partial S_{x}=0$.

The only remaining case in the proof of Theorem 4.8 is when $n-d=2$, and the poles $Z$ are of dimension 1 .

If $\operatorname{dim}_{\mathcal{H}}(Z)=1$, then $Z$ is the image of a one-dimensional rectifiable set under $\Pi_{2}$, since $\mathcal{L}^{1}$-a.e. slices by the radius of the transverse $h$-cone $\varphi$ are rectifiable, by slicing theory $[2,4.3 .1]$. Of course, then any radius would yield a rectifiable section, since $\varphi$ is an $h$-cone.

Let $\lambda_{i} \downarrow 0$ be a sequence of stretches so that $S_{i}:=S_{\lambda_{i}}$ converges to $\psi \in$ $\operatorname{Cart}\left(B_{r} \times F\right)$. If $U_{m}: B_{r} \rightarrow F$ is a sequence of smooth graphs converging to $S$, then for some subsequence $\left\{i_{m}\right\}$ the sequence of stretches $\left\{U_{m, i_{m}}\right\}:=$ $\left\{\left(\nu_{1 / \lambda_{i_{m}}}\right)_{\#}\left(U_{m}\right)\right\}$ will converge in $\operatorname{Cart}\left(B_{r} \times S\right)$ to $\psi$.

$Z$ is clearly the limit of the images of smooth curves $C_{m}: S^{1} \rightarrow F$, which are the curves $u_{m \mid \partial B_{r}^{2}}$ where $u_{m}:=U_{m \mid L^{\perp} \cap B_{r}}$ converges to the transverse $h$ cone $\varphi$. Since $Z$ has no boundary, then, it is the $\Pi_{2}$-image of a continuous curve in $S^{1} \times F$ (not necessarily a continuous graph, but the curve has a continuous parameterization).

Assume, without loss of generality, that the radius of the domain is 1 . Since the current is an $h$-cone, the radius is irrelevant.

If the image $Z$ is a closed curve, then $F-Z$ will have a strong deformation retraction to a compact set $K$ in $F$. $Z$ will topologically be a wedge of circles. However, the maps $u_{m}: D^{2} \rightarrow F$ satisfy $u_{m \mid \partial D^{2}}=C_{m}$, so each curve is nullhomotopic and for $m$ sufficiently large the images of $u_{m}$ must miss $K$, since $K \cap \operatorname{Supp}(Z)=\varnothing$, and $Z$ is the set of limit points of the images of $u_{m} . K$ can be chosen, if $Z$ is a closed curve, to be the complement of a smoothly immersed solid torus. If $Z$ is homotopy-equivalent to a circle, then $G:=F-K$ is an embedded solid torus, $G:=S^{1} \times D^{j-1}$. and if $Z$ has trivial homotopy type, then $G$ can be taken to be a cylinder $I \times D^{j-1}$. The general case would be that $Z$ has the homotopy type of a wedge of more than one circle. The other cases follow similarly. In this case, since $Z$ is a limit of images of graphs over 
the circle, there is an immersed solid torus with image $G$ through which the graphs $u_{m}$ factor, for $m$ sufficiently large, since the various self-intersections of $u_{m}$ have a neighborhood in $F$ whose inverse image under $u_{m}$ is disconnected, corresponding to different branches of the immersion $\tau: S^{1} \times D^{j-1} \rightarrow G$. Then $u_{m}(x)=\tau\left(a_{m}(x), b_{m}(x)\right) \in G$. Since $u_{m}$ is a map from the disk, $a_{m}(x)$ admits a lift $A_{m}: D^{2} \rightarrow \mathbb{R}$, with $\pi: \mathbb{R} \rightarrow S^{1}$ the usual projection, so that $a_{m}=$ $\pi A_{m}$. Since the domain of $A_{m}$ is compact, and $A_{m}$ converge in $\operatorname{Cart}\left(D^{2} \times\right.$ $F$ ), we may assume there is a $C_{m}>0$ sufficiently large so that $A_{m}: D^{2} \rightarrow$ $\left[0, C_{m}\right]$, with $C_{m} \rightarrow C$, taking a subsequence if necessary, since $\left\{C_{m}\right\}$ is not $a$ priori convergent. Assume that each $C_{m}$ is the smallest possible such constant. Change, if necessary, the immersion of $S^{1} \times D^{j-1}$ so that, for some $\delta>0$, in a neighborhood of all points $\tau\left(a_{m}(x), b_{m}(x)\right)$ where $a_{m}(x) \in\left[C_{m}-\delta, C_{m}\right]$, the coordinate distributions $\tau_{*}\left(T_{*}\left(S^{1}\right)\right)$ and $\tau_{*}\left(T_{*}\left(D^{j-1}\right)\right)$ are orthogonal. There are no obstructions to the choice of such a neighborhood, for small enough $\delta$, since the set of all such points is a compact subset of the open set $G$, which may be assumed to be contained within a single coordinate patch.

Define, for each $\varepsilon>0, \varepsilon<\delta$, a sequence $J_{m, \varepsilon}: D^{2} \rightarrow F$ by

$$
J_{m, \varepsilon}(x)= \begin{cases}\tau\left(\pi\left(A_{m}(x)\right), b_{m}(x)\right), & \text { if } A_{m}(x)<C_{m}-\varepsilon, \\ \tau\left(\pi\left(C_{m}+\left(A_{m}(x)-C_{m}\right)\|x\|, b_{m}(x)\right),\right. & \text { if } A_{m}(x) \geq C_{m}-\varepsilon .\end{cases}
$$

FiguRE 4.3: $\tau\left(S^{1} \times D^{j-1}\right)$, with $u_{m}(x)$ and $J_{m, \varepsilon}(x)$

Note that the original boundary conditions still hold. The question is to show that this current has less $\mathcal{V}_{\lambda_{i_{m}}}$-energy. However, since $u_{m} \rightarrow \varphi$ in 
$\operatorname{Cart}\left(D^{2} \times F\right)$, if we can show that the $\mathcal{V}_{\lambda_{i_{m}}}$-energy of the transverse $h$-cone $\varphi$ is greater than the $\mathcal{V}_{\lambda_{i_{m}}}$-energy of the similarly modified currents $\varphi_{\varepsilon}$ defined by

$$
\varphi_{\varepsilon}(x)= \begin{cases}\tau(\pi(A(x)), b(x)), & \text { if } A(x)<C-\varepsilon, \\ \tau(\pi(C+(A(x)-C)\|x\|, b(x))), & \text { if } A(x) \geq C-\varepsilon,\end{cases}
$$

where $\varphi_{\varepsilon}(x)=\tau(\pi(A(x)), b(x))$. Note that writing $\varphi$ as regular on $D^{2}-\{0\}$ is not inaccurate, since the singularities of $\psi$ are on the subspace $L$, and so $\varphi$ (and $S_{\lambda}$ ) is regular except along the intersection with $L$, which occurs only at the origin in $D^{2}$.

Figure 4.4: A slice of the $\mathcal{V}_{\lambda_{i_{m}}}$-energy of $\varphi$ in comparison with that of $\varphi_{\varepsilon}$

Now, the region $W \subset D^{2}$ where $A(x) \geq C-\varepsilon$ will be the interior of a cone (or several), subtending an angle $\theta_{1}$. We can replace the graph $\tau(\pi(A(x)), b(x))$ by a curve $\gamma(\theta)$ in $F$, since the fiber over $Z$ is one-dimensional and $\varphi$ is an $h$ cone (and is regular except at 0 ). The $\mathcal{V}_{{\lambda_{i}}_{m}}$-energy of that cone can be explicitly computed as

$$
\int_{0}^{1} \int_{0}^{\theta_{1}} \sqrt{\left(\frac{d \gamma}{d \theta}\right)^{2}+\lambda_{i_{m}}{ }^{2} r^{2}} d \theta d r
$$

as suggested by Figure 4.4. Similarly, the $\mathcal{V}_{\lambda_{i_{m}}}$-energy of the comparison graph $\varphi_{\varepsilon}$, over that same cone $W$, is similarly computed as

$$
\int_{0}^{1} \int_{0}^{\theta_{1}} \sqrt{r^{2}\left(\frac{d \gamma}{d \theta}\right)^{2}+\lambda_{i_{m}}{ }^{2} r^{2}} \sqrt{{\lambda_{i_{m}}{ }^{2}+(C-A(\theta))^{2}}^{2}} d \theta d r
$$


where $A(\theta)=A(x)$, since on the cone $A$ only varies with $\theta$.

The excess amount of $\mathcal{V}_{\lambda_{i_{m}}}$-energy by the transverse $h$-cone $\varphi$ is then measured as

$$
\int_{0}^{1} \int_{0}^{\theta_{1}} \sqrt{\left(\frac{d \gamma}{d \theta}\right)^{2}+\lambda_{i_{m}}{ }^{2} r^{2}}-\sqrt{r^{2}\left(\frac{d \gamma}{d \theta}\right)^{2}+\lambda_{i_{m}}{ }^{2} r^{2}} \sqrt{{\lambda_{i_{m}}{ }^{2}+(C-A(\theta))^{2}}^{2}} d \theta d r
$$

This integral can in turn best be interpreted when $\lambda_{i_{m}}$ is taken as 0 (for $m$ large, it will approach 0 ), where the integral becomes:

$$
\int_{0}^{1} \int_{0}^{\theta_{1}}\left|\frac{d \gamma}{d \theta}\right|(1-r\|C-A(\theta)\|) d \theta d r
$$

which is clearly positive, and in fact is at least $2 \varepsilon-\varepsilon^{2}$, so the transverse $h$-cone has more energy than $\varphi_{\varepsilon}$. Thus, for $\lambda_{i_{m}}$ sufficiently small ( $m$ large enough), $J_{m, \varepsilon}$ will have smaller $\mathcal{V}_{\lambda_{i_{m}}}$-energy than $u_{m}$, by an amount bounded away from 0 as $m \rightarrow \infty$, for fixed $\varepsilon$.

This construction can be made for all slices of $B_{r}$ by translates of $L^{\perp}$. For a large enough $m$, the pole of the slices at $x \neq 0$ in $L$ will not be exactly on $L$, but the construction above can of course still be carried through. To meet the boundary conditions, simply take $\varepsilon$ to 0 as the pole point of the transverse slice approaches $\partial B_{r}$. Thus the original $U_{m, i_{m}}$ cannot have been $\mathcal{V}_{\lambda_{i_{m}}}$-minimizing.

Since this was the last possibility under the assumption that the dimension of the singular set was larger than $n-3$, this completes the proof of Theorem 4.9 .

\section{REFERENCES}

[1] F. J. Almgren, JR., Q-valued functions minimizing Dirichlet's integral and the regularity of area minimizing rectifiable currents up to codimension two, Bull. Amer. Math. Soc. 8 (1983), 327-328.

[2] H. Federer, Geometric Measure Theory, Springer-Verlag, 1969.

[3] M. Giaquinta, G. Modica 83 JiŘi Souček, Cartesian currents and variational problems for mappings into spheres, Ann. Scuola Norm. Sup. Pisa 16 (1989), 393-485.

[4] Cartesian currents, weak diffeomorphisms and existence theorems in nonlinear elasticity, Arch. Rational Mech. Anal. 106 (1989), 97-159.

[5] H. Gluck \& W. Ziller, On the volume of a unit vector field on the three-sphere, Comment. Math. Helv. 61 (1986), 177-192.

[6] R. Hardt 85 L. Simon, Seminar on Geometric Measure Theory, Birkhäuser Boston, 1986.

[7] D. L. Johnson, Volume of flows, Proc. Amer. Math. Soc. 104 (1988), 923-931.

[8] - Kähler submersions and holomorphic connections, J. Differential Geom. 15 (1980), 71-79. 
[9] G. LAwler, A sufficient condition for a cone to be area-minimizing, Mem. Amer. Math. Soc. 91 (1991).

[10] J. D. Moore \& R. Schlafly, On equivariant isometric embeddings, Math. Z. 173 (1980), 119-133.

[11] F. Morgan, Geometric Measure Theory, A Beginner's Guide, Academic Press, New York, 1988.

[12] S. Pedersen, Volumes of vector fields on spheres, Trans. Amer. Math. Soc. 336 (1993), 69-78.

[13] T. SASAKI, On the differential geometry of tangent bundles of Riemannian manifolds, Tôhoku Math. J. 10 (1958), 338-354.

[14] R. Schoen, The role of harmonic mappings in rigidity and deformation problems., Complex Geometry, Osaka International Conference, 1990, Lecture Notes in Pure and Applied Mathematics, 143 (Gen Komatsu and Yusuke Sakane, eds.), Marcel Dekker, 1993 pages $179-200$, pp. 179-200.

[15] Leon Simon, Lectures on geometric measure theory, Proc. Centre Math. Anal. Austral. Nat. Univ. 3 (1983).

DAVID L. JOHNSON

Department of Mathematics

Lehigh University

Bethlehem, Pennsylvania 18015-3174

E-MAIL: dlj0@lehigh.edu
Penelope Smith

Department of Mathematics

Lehigh University

Bethlehem, Pennsylvania 18015-3174

E-MAIL:ps02@lehigh.edu

Received: November 29th, 1993; revised: March 30th, 1995. 